\title{
Wireless Communications with Reconfigurable Intelligent Surface: Path Loss Modeling and Experimental Measurement
}

\author{
Wankai Tang, Ming Zheng Chen, Xiangyu Chen, Jun Yan Dai, Yu Han, \\ Marco Di Renzo, Yong Zeng, Shi Jin, Qiang Cheng, and Tie Jun Cui
}

\begin{abstract}
Reconfigurable intelligent surfaces (RISs) comprised of tunable unit cells have recently drawn significant attention due to their superior capability in manipulating electromagnetic waves. In particular, RIS-assisted wireless communications have the great potential to achieve significant performance improvement and coverage enhancement in a cost-effective and energy-efficient manner, by properly programming the reflection coefficients of the unit cells of RISs. In this paper, free-space path loss models for RIS-assisted wireless communications are developed for different scenarios by studying the physics and electromagnetic nature of RISs. The proposed models, which are first validated through extensive simulation results, reveal the relationships between the free-space path loss of RIS-assisted wireless communications and the distances from the transmitter/receiver to the RIS, the size of the RIS, the near-field/far-field effects of the RIS, and the radiation patterns of antennas and unit cells. In addition, three fabricated RISs (metasurfaces) are utilized to further corroborate the theoretical findings through experimental measurements conducted in a microwave anechoic chamber. The measurement results match well with the modeling results, thus validating the proposed free-space path loss models for RIS, which may pave the way for further theoretical studies and practical applications in this field.
\end{abstract}

Index Terms-Path loss, reconfigurable intelligent surface, metasurface, intelligent reflecting surface, large intelligent surface, wireless propagation measurements.

\section{INTRODUCTION}

It is foreseen that the commercial service of the fifthgeneration $(5 \mathrm{G})$ of mobile communications will be launched

This work was supported in part by the National Key Research and Development Program of China under Grant 2018YFA0701602, Grant 2018YFA0701904, Grant 2017YFA0700201, Grant 2017YFA0700202, and Grant 2017YFA0700203, in part by the National Science Foundation of China (NSFC) for Distinguished Young Scholars under Grant 61625106, and in part by NSFC under Grant 61941104, Grant 61722106, and Grant 61731010. The work of Yong Zeng was supported in part by the Research Fund of National Mobile Communications Research Laboratory, Southeast University (No.2020B06), and in part by the Fundamental Research Funds for the Central Universities under grant number 3204002004A2. The work of M. Di Renzo was supported in part by the European Commission through the H2020 ARIADNE project under grant agreement 675806. (Corresponding authors: Shi Jin; Qiang Cheng; Tie Jun Cui.)

W. Tang, X. Chen, Y. Han, Y. Zeng, and S. Jin are with the National Mobile Communications Research Laboratory, Southeast University, Nanjing 210096, China (e-mail: tangwk@ @eu.edu.cn; chenxy19@ seu.edu.cn; hanyu@ seu.edu.cn; yong_zeng@seu.edu.cn; jinshi@seu.edu.cn).

M. Z. Chen, J. Y. Dai, Q. Cheng, and T. J. Cui are with the State Key Laboratory of Millimeter Waves, Southeast University, Nanjing 210096, China (e-mail: mingzhengc@ @eu.edu.cn; junyandai@ seu.edu.cn; qiangcheng@seu.edu.cn; tjcui@seu.edu.cn).

M. Di Renzo is with Université Paris-Saclay, CNRS, CentraleSupélec, Laboratoire des Signaux et Systèmes, 3 Rue Joliot-Curie, 91192 Gif-surYvette, France. (e-mail: marco.direnzo@centralesupelec.fr). on a worldwide scale from 2020. Massive multiple-input multiple-output (MIMO) base stations (BSs) with full-digital transceivers are being commercially deployed and tested in several countries [1]. Meanwhile, the success of millimeter wave (mmWave) trials and testbeds across the world ensures that mmWave wireless communications will be realized by 2020 [2]. As the two key technologies of the 5G physical layer, massive MIMO and mmWave will significantly increase the network capacity and resolve the spectrum shortage issue in current cellular communication systems [3]. On the other hand, the International Telecommunication Union (ITU) predicted that the exponential growth of the global mobile data traffic will continue, which will reach 5 zettabytes $(\mathrm{ZB}, 1 \mathrm{ZB}=$ $10^{21}$ Bytes) per month by 2030 [4]. The ever-increasing traffic demands are driven by emerging data-intensive applications, such as virtual reality, augmented reality, holographic projection, autonomous vehicle, tactile Internet, to name a few, which can hardly be offered by $5 \mathrm{G}$. Therefore, there has been increasing research interest from both academia and industry towards the sixth-generation $(6 \mathrm{G})$ of mobile communications.

Looking forward to future $6 \mathrm{G}$ wireless communication technologies, one possibility is to further extend the spectrum to terahertz $(\mathrm{THz})$ band $[5]$ and the antenna array scale to ultra-massive MIMO (UM-MIMO) [6] [7], which can further boost the spatial diversity and expand the available spectrum resources. Besides, artificial intelligence (AI), orbital angular momentum (OAM) multiplexing, visible-light communications (VLC), blockchain-based spectrum sharing, quantum computing, etc., to name just a few, are being actively discussed as potential enabling technologies for $6 \mathrm{G}$ mobile communication systems [8]. Furthermore, reconfigurable intelligent surfaces (RISs) 1 which are an emerging technology to manipulate electromagnetic waves, have drawn significant attention due to their capability in tailoring electromagnetic waves across a wide frequency range, from microwave to visible light. RIS technology is enabled by metasurfaces comprised of subwavelength unit cells with tunable electromagnetic responses, such as amplitude, phase, polarization and frequency, which can be controlled by external signals in a real-time reconfigurable manner during the light-matter interaction [9]-[11]. Their programmable electromagnetic properties make RISs especially appealing for wireless communications.

\footnotetext{
${ }^{1}$ Also known as intelligent reflecting surfaces (IRSs) and passive large intelligent surfaces (LISs) in the literature.
} 
Recently, we have witnessed fast growing research effort on wireless communication using RISs. RISs have been utilized to realize new wireless transceiver architectures in [12]-[15], which may bring a paradigm-shift on the transceiver design and reduce the hardware cost of future wireless communication systems. Moreover, RISs can artificially shape the electromagnetic wave propagation environment [16]. In current wireless communication systems, the wireless environment, i.e., the physical objects that influence the propagation of the electromagnetic waves, is uncontrollable. During the research and design processes of wireless communication systems, one can only design transceivers, signal processing algorithms, and transmission protocols to adapt to the radio environment. RISs, on the other hand, may in principle make the wireless environment controllable and programmable, thus bringing unprecedented new opportunities for enhancing the performance of wireless communication systems [17] [18].

Against this background, various RIS-assisted wireless communication systems have recently been studied, by providing cost-effective and energy-efficient solutions that offer performance improvements and coverage enhancement in wireless networks [19]. For example, [20] showed that, by jointly optimizing active and passive beamforming in an RISassisted wireless network, the energy consumption and the coverage performance can be significantly improved. The joint beamforming design was also investigated for improving the physical layer security [21] [22]. The authors of [23][25] optimized the achievable rate of RIS-assisted systems in different communication scenarios. The energy efficiency of RIS-based downlink multi-user MISO systems was maximized in [26] [27]. Energy harvesting performance, ergodic spectral efficiency, symbol error probability, and outage probability of RIS-assisted wireless communications were derived and optimized in [28]-[31]. The comparison with relays was carried out in [32]. The impact of hardware imperfections, like discrete phase-shift and phase-dependent amplitude, were studied in [29] [33] [34]. Channel estimation for RIS-assisted systems was discussed in [35]-[37]. The impact of channel estimation and feedback overhead was recently analyzed in [38].

However, the major limitation of existing research on RISassisted wireless communications is the lack of tractable and reliable physical and electromagnetic models for the RISs. Most of the existing research works are based on simplistic mathematical models that regard the RIS as a diagonal matrix with phase shift values. The responses of the RISs to the radio waves have not yet been extensively studied from the physics and electromagnetic point of view, which may lead to relatively simplified algorithm designs and performance predictions. To the best of the authors' knowledge, in particular, there is no experimentally validated path loss model for RISassisted wireless communications, even in the simple freespace propagation environment. The path loss models used in most existing works do not consider physical factors such as the size of the RISs, and the near-field/far-field effects of the RISs. Therefore, channel measurements are urgently needed, which may provide authentic information on the path loss of RIS-assisted wireless communications. Motivated by these conditions, we develop free-space path loss models for RISassisted wireless communications in different scenarios based on the physics and electromagnetic nature of the RISs. We validate them by using numerical simulations, and we also conduct channel measurements by using fabricated RISs in an anechoic chamber to practically corroborate our findings. In particular, our main contributions are summarized as follows:

1) We introduce a system model for RIS-assisted wireless communications from the perspective of electromagnetic theory, which takes into account important physical factors like the physical dimension of the RISs, and the radiation pattern of the unit cells. A general formula is derived to characterize the free-space path loss of RISassisted wireless communications.

2) Based on the derived general formula, we propose three free-space path loss models for RIS-assisted wireless communications, which capture three relevant scenarios and unveil the relationships between the free-space path loss of RIS and the distances from the transmitter/receiver to the RIS, the size of the RIS, the nearfield/far-field effects of the RIS, as well as the radiation patterns of antennas and unit cells.

3) We report the world's first experimental results that assess and validate the path loss of RIS-assisted wireless communications. Three different RISs (metasurfaces) of various size are utilized in the measurements. The measurement results are shown to be in good agreement with the modeling results. Besides, the power consumption of the RISs and the effect of the incident angle are also measured and discussed.

\section{PRELIMINARIES}

This section reviews several basic concepts, which will be used in the free-space path loss modeling of RIS-assisted wireless communications in the next section.

\section{A. Far Field and Near Field}

When a transmitter is sufficiently far away from an antenna array, the spherical wave generated by the transmitter can be approximately regarded as a plane wave at the antenna array side. Specifically, when the maximum phase difference of the received signal on the antenna array does not exceed $\frac{\pi}{8}$, the transmitter is in the far field of the antenna array [39]. Based on this assumption, the boundary of the far field and the near field of the antenna array is defined as $L=\frac{2 D^{2}}{\lambda}$, where $L, D$ and $\lambda$ denote the distance between the transmitter and the center of the antenna array, the largest dimension of the antenna array and the wave length of the signal, respectively [39]. It's worth noting that when the transmitter is replaced by a receiver, the above definition remains the same due to the reciprocity of the antenna array. We will use the same definition of the far field and near field for RISs in the next section. When the distance between the transmitter/receiver and the center of the RIS is less than $\frac{2 D^{2}}{\lambda}$, 


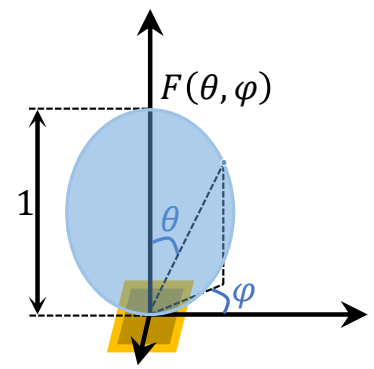

Fig. 1. Diagram of normalized power radiation pattern.

the transmitter/receiver are considered to be in the near field ${ }^{2}$ of the RIS. Otherwise, they are in the far field of the RIS.

\section{B. Power Radiation Pattern and Gain}

The power radiation pattern defines the variation of the power radiated or received by an antenna as a function of the direction away from the antenna, which allows us to visualize where the antenna transmits or receives the maximum power. The normalized power radiation pattern can be written as a function $F(\theta, \varphi)$ in the spherical coordinate system as shown in Fig. 1. where $\theta$ and $\varphi$ are the elevation and azimuth angles from the antenna to a certain transmitting/receiving direction. An example of normalized power radiation pattern is the following [40]

$$
F(\theta, \varphi)=\left\{\begin{aligned}
\cos ^{3} \theta & \theta \in\left[0, \frac{\pi}{2}\right], \varphi \in[0,2 \pi] \\
0 & \theta \in\left(\frac{\pi}{2}, \pi\right], \varphi \in[0,2 \pi]
\end{aligned}\right.
$$

The specific normalized power radiation pattern represented by (1) is only a function of the elevation angle $\theta$ and is maximized when $\theta=0$, which indicates that the corresponding antenna has maximum gain towards the direction of $\theta=0$. The term antenna gain describes how much power is transmitted or received in the direction of peak radiation relative to that of an isotropic antenna, which, by assuming $100 \%$ antenna efficiency, can be written as [40]

$$
\text { Gain }=\frac{4 \pi}{\int_{\varphi=0}^{2 \pi} \int_{\theta=0}^{\pi} F(\theta, \varphi) \sin \theta d \theta d \varphi} .
$$

Consider the antenna with power radiation pattern described by (1) as an example, its gain is equal to $8(9.03 \mathrm{dBi})$ under the assumption of $100 \%$ radiation efficiency, which means that the power transmitted or received in the direction of peak radiation $(\theta=0)$ is $9.03 \mathrm{~dB}$ higher than that of an isotropic antenna. In the next section, we will use the definition of normalized power radiation pattern not only for the antennas of the transmitter and receiver, but also for the unit cells of RIS

\footnotetext{
${ }^{2}$ We assume that the distance between the transmitter/receiver and each unit cell of the RIS is larger than $5 \lambda$ throughout this paper [40], which is regarded as the lower bound of the near field of the RIS in this paper. This assumption ensures that for each unit cell of the RIS, we can consider only the unit cell's far field component: the incident/reflected power of each unit cell follows the inverse square law [41]. As a result, the near field range of the RIS is defined as between $5 \lambda$ and $\frac{2 D^{2}}{\lambda}$ in this paper. It is worth noting that the RIS used for assisting wireless communication usually satisfy that $\frac{2 D^{2}}{\lambda}$ is greater than $5 \lambda$, ie., $D>1.58 \lambda$. If an RIS is too small to support $\frac{2 D^{2}}{\lambda}>5 \lambda$, then this RIS only has far-field region where the distance $>5 \lambda$.
}

in the free-space path loss modeling of RIS-assisted wireless communication ${ }_{3}^{3}$

\section{Free-Space Path Loss Modeling of RIS-ASSISTED WIRELESS COMMUNICATIONS}

In this section, we describe the overall system model and develop the free-space path loss model for RIS-assisted wireless communications in different scenarios.

\section{A. System Model}

We consider a general RIS-assisted single-input singleoutput (SISO) wireless communication system as shown in Fig. 2 Since this paper aims to study the free-space path loss modeling of RIS-assisted wireless communications, the direct link between the transmitter and the receiver is ignored in the rest of the paper $1^{4}$. The RIS is placed in the $x-y$ plane of a Cartesian coordinate system, and the geometric center of the RIS is aligned with the origin of the coordinate system. Let $N$ and $M$ denote the number of rows and columns of the regularly arranged unit cells of the RIS, respectively. The size of each unit cell along the $\mathrm{x}$ axis is $d_{x}$ and that along the y axis is $d_{y}$, which are usually of subwavelength scale within the range of $\frac{\lambda}{10}$ and $\frac{\lambda}{2} \cdot F(\theta, \varphi)$ is the normalized power radiation pattern of the unit cell, which reveals the dependence of the incident/reflected power density of the unit cell on the incident/reflected angle. $G$ is the gain of the unit cell, which is defined by (2) and is only related to the normalized power radiation pattern $F(\theta, \varphi)$ of the unit cell. $U_{n, m}$ denotes the unit cell in the $n^{t h}$ row and $m^{\text {th }}$ column with the programmable reflection coefficient $\Gamma_{n, m}$. The center position of $U_{n, m}$ is $\left(\left(m-\frac{1}{2}\right) d_{x},\left(n-\frac{1}{2}\right) d_{y}, 0\right)$, where $m \in\left[1-\frac{M}{2}, \frac{M}{2}\right]$ and $n \in\left[1-\frac{N}{2}, \frac{N}{2}\right]$, assuming that both of $N$ and $M$ are even numbers. We use the symbols $d_{1}$, $d_{2}, \theta_{t}, \varphi_{t}, \theta_{r}$ and $\varphi_{r}$ to represent the distance between the transmitter and the center of the RIS, the distance between the receiver and the center of the RIS, the elevation angle and the azimuth angle from the center of the RIS to the transmitter, the elevation angle and the azimuth angle from the center of the RIS to the receiver, respectively. Let $r_{n, m}^{t}, r_{n, m}^{r}$, $\theta_{n, m}^{t}, \varphi_{n, m}^{t}, \theta_{n, m}^{r}$ and $\varphi_{n, m}^{r}$ represent the distance between the transmitter and $U_{n, m}$, the distance between the receiver and $U_{n, m}$, the elevation angle and the azimuth angle from $U_{n, m}$ to the transmitter, the elevation angle and the azimuth angle from $U_{n, m}$ to the receiver, respectively.

As shown in Fig. 2, the transmitter emits a signal to the RIS with power $P_{t}$ through an antenna with normalized power radiation pattern $F^{t x}(\theta, \varphi)$ and antenna gain $G_{t}$. The signal is reflected by the RIS and received by the receiver with normalized power radiation pattern $F^{r x}(\theta, \varphi)$ and antenna

\footnotetext{
${ }^{3}$ We use (1) to match the normalized power radiation pattern of the unit cell of the RIS in this paper, because the -3dB main lobe width of the unit cell of the three utilized RISs in Section IV and V is about $75^{\circ}\left(\cos ^{3}\left(\frac{75^{\circ}}{2}\right)=0.5\right)$. The form $\cos ^{\alpha}$ can be used to match the normalized power radiation pattern of different unit cell and antenna designs with appropriate $\alpha[40]$.

${ }^{4}$ If the direct link exists, the received signal becomes the sum of the signal reflected from the RIS and the signal transmitted from the direct link. Since the channel model of the direct link has already been well studied [42], this work focuses on the RIS-assisted link and aims to provide its free-space path loss models in different scenarios.
} 


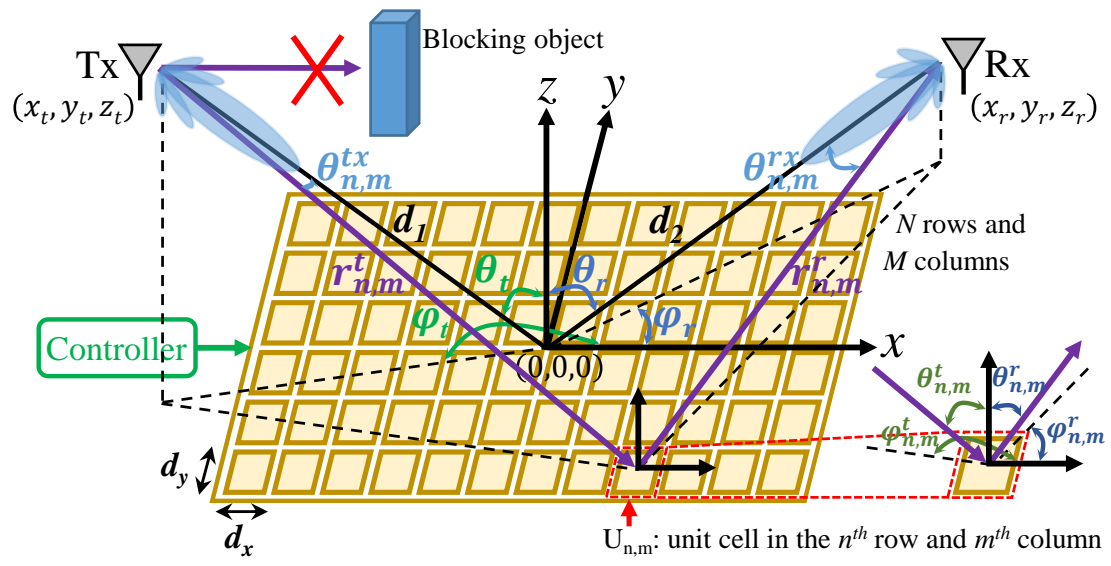

Fig. 2. RIS-assisted wireless communication without the direct path between the transmitter and the receiver.

gain $G_{r}$. Let $\theta_{n, m}^{t x}, \varphi_{n, m}^{t x}, \theta_{n, m}^{r x}$ and $\varphi_{n, m}^{r x}$ represent the elevation angle and the azimuth angle from the transmitting antenna to the unit cell $U_{n, m}$, and the elevation angle and the azimuth angle from the receiving antenna to the unit cell $U_{n, m}$, respectively. We assume that the polarization of the transmitter and receiver are always properly matched, even after the transmitted signal is reflected by the RIS.

The following key result presents the connection between the received signal power of the receiver and various parameters described above in RIS-assisted wireless communication systems.

Theorem 1: The received signal power in RIS-assisted wireless communications is as follows

$$
\begin{aligned}
P_{r} & =P_{t} \frac{G_{t} G_{r} G d_{x} d_{y} \lambda^{2}}{64 \pi^{3}} \\
& \times\left|\sum_{m=1-\frac{M}{2}}^{\frac{M}{2}} \sum_{n=1-\frac{N}{2}}^{\frac{N}{2}} \frac{\sqrt{F_{n, m}^{\text {combine }}} \Gamma_{n, m}}{r_{n, m}^{t} r_{n, m}^{r}} e^{\frac{-j 2 \pi\left(r_{n, m}^{t}+r_{n, m}^{r}\right)}{\lambda}}\right|^{2},
\end{aligned}
$$

where $F_{n, m}^{\text {combine }}=F^{t x}\left(\theta_{n, m}^{t x}, \varphi_{n, m}^{t x}\right) F\left(\theta_{n, m}^{t}, \varphi_{n, m}^{t}\right) F\left(\theta_{n, m}^{r}\right.$, $\left.\varphi_{n, m}^{r}\right) F^{r x}\left(\theta_{n, m}^{r x}, \varphi_{n, m}^{r x}\right)$, which accounts for the effect of the normalized power radiation patterns on the received signal power.

Proof: See Appendix A.

Theorem 1 reveals that the received signal power is proportional to the transmitted signal power, the gains of the transmitting/receiving antennas, the gain of the unit cell, the size of the unit cell, the square of the wave length. In addition, Theorem 1 also indicates that the received signal power is related to the normalized power radiation patterns of the transmitting/receiving antennas and unit cells, the reflection coefficients of the unit cells, and the distances between the transmitter/receiver and the unit cells. However, their specific relationships are not that straightforward, which needs further analysis and discussions in the rest of this paper. Theorem 1 also shows that RIS-assisted wireless communication systems are transmitter-receiver reciprocal, since the same signal power can be received if the roles of the transmitter and the receiver are exchanged, which is an especially important property in the uplink and downlink design of time division duplexing (TDD) wireless communication systems.

In this paper, we develop free-space path loss models for
RIS-assisted wireless communications in different scenarios, which can be classified into two categories: RIS-assisted beamforming and RIS-assisted broadcasting as shown in Fig. 3 In the RIS-assisted beamforming scenario, the received signal power is maximized for a single specific user. On the other hand, in the RIS-assisted broadcasting scenario, the signal evenly covers all users in a specific area. We regard (3) as a general formula. As each considered scenario depends on the design of the reflection coefficients of the unit cells, as well as the near-field/far-field effects of the RIS, (3) will be further discussed for different cases to draw insights on the free-space path loss of RIS-assisted wireless communications.

\section{B. Far Field Beamforming Case}

The signals reflected by all the unit cells of the RIS to the receiver can be aligned in phase to enhance the received signal power, which makes RISs especially appealing for beamforming applications. Therefore, it is important to study the free-space path loss in RIS-assisted beamforming scenario. The case where both of the transmitter and receiver are in the far field of the RIS is first discussed as follows.

Proposition 1: Assume that the directions of peak radiation of both the transmitting and receiving antennas point to the center of the RIS, and all the unit cells of the RIS share the same reflection coefficient $\Gamma_{n, m}=A e^{j \phi}$. The received signal power in the far field case can be written as

$$
\begin{aligned}
P_{r}= & P_{t} \frac{G_{t} G_{r} G M^{2} N^{2} d_{x} d_{y} \lambda^{2} F\left(\theta_{t}, \varphi_{t}\right) F\left(\theta_{r}, \varphi_{r}\right) A^{2}}{64 \pi^{3} d_{1}^{2} d_{2}^{2}} \\
& \times \mid \frac{\operatorname{sinc}\left(\frac{M \pi}{\lambda}\left(\sin \theta_{t} \cos \varphi_{t}+\sin \theta_{r} \cos \varphi_{r}\right) d_{x}\right)}{\operatorname{sinc}\left(\frac{\pi}{\lambda}\left(\sin \theta_{t} \cos \varphi_{t}+\sin \theta_{r} \cos \varphi_{r}\right) d_{x}\right)} \\
& \times\left.\frac{\operatorname{sinc}\left(\frac{N \pi}{\lambda}\left(\sin \theta_{t} \sin \varphi_{t}+\sin \theta_{r} \sin \varphi_{r}\right) d_{y}\right)}{\operatorname{sinc}\left(\frac{\pi}{\lambda}\left(\sin \theta_{t} \sin \varphi_{t}+\sin \theta_{r} \sin \varphi_{r}\right) d_{y}\right)}\right|^{2} .
\end{aligned}
$$

If $\theta_{r}=\theta_{t}$ and $\varphi_{r}=\varphi_{t}+\pi$, (4) is maximized as

$P_{r}^{\max }=\frac{G_{t} G_{r} G M^{2} N^{2} d_{x} d_{y} \lambda^{2} F\left(\theta_{t}, \varphi_{t}\right) F\left(\theta_{r}, \varphi_{r}\right) A^{2}}{64 \pi^{3} d_{1}{ }^{2} d_{2}{ }^{2}} P_{t}$

The path loss corresponding to $[5]$ is

$P L_{\text {farfield }}^{\text {beam }}=\frac{64 \pi^{3}\left(d_{1} d_{2}\right)^{2}}{G_{t} G_{r} G M^{2} N^{2} d_{x} d_{y} \lambda^{2} F\left(\theta_{t}, \varphi_{t}\right) F\left(\theta_{r}, \varphi_{r}\right) A^{2}}$. 


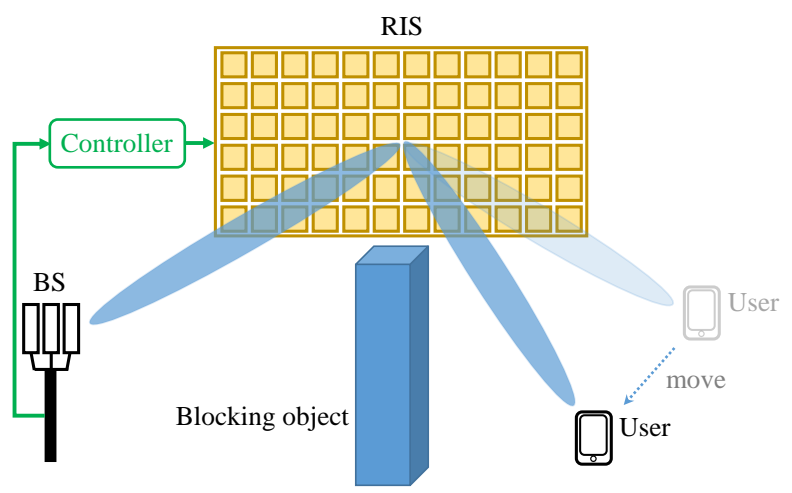

(a)

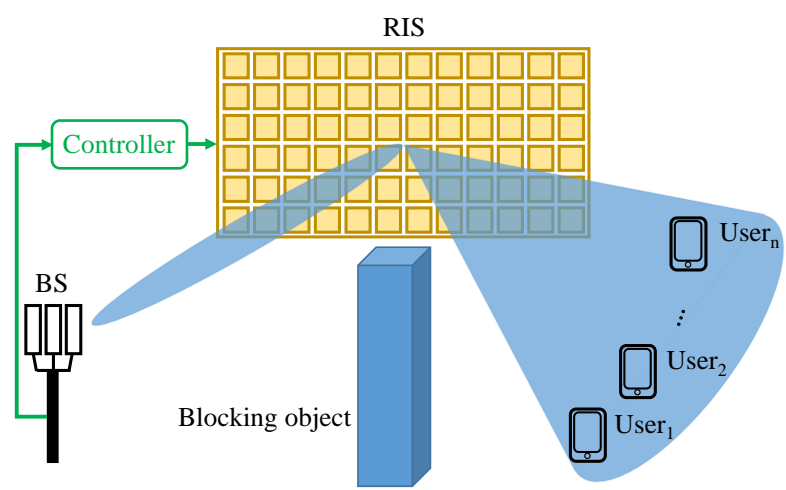

(b)

Fig. 3. Two scenarios of RIS-assisted wireless communications. (a) RIS-assisted beamforming. (b) RIS-assisted broadcasting.

Proof: See Appendix B.

Proposition 1 is more insightful compared with the general formula (3) in Theorem 1 and equation (4) is referred to as the far-field formula. Proposition 1 reveals that the freespace path loss of RIS-assisted wireless communications is proportional to $\left(d_{1} d_{2}\right)^{2}$ in the far field case. The free-space path loss is also related to the unit cells' normalized power radiation pattern $F(\theta, \varphi)$, which is fixed once the RIS is designed and fabricated. Furthermore, when the reflection coefficients of all the unit cells are the same, the RIS performs specular reflection, that is, the incident signal is mainly reflected towards the mirror direction $\left(\theta_{r}=\theta_{t}\right.$ and $\left.\varphi_{r}=\varphi_{t}+\pi\right)$.

The above analysis assumes that all the unit cells share the same reflection coefficient. The design of $\Gamma_{n, m}$ is discussed in the following proposition, which enables the RIS to beamform the reflected signal to the receiver in any desired direction $\left(\theta_{\text {des }}, \varphi_{\text {des }}\right)$ through intelligent reflectior ${ }^{5}$

Proposition 2: Assume that the directions of peak radiation of both the transmitting and receiving antennas point to the center of the RIS, and the reflection coefficients of all the unit cells share the same amplitude value $A$ and different phase shift $\phi_{n, m}\left(\Gamma_{n, m}=A e^{j \phi_{n, m}}\right)$. The received signal power through intelligent reflection in the far field case can be written as

$$
\begin{aligned}
P_{r}= & P_{t} \frac{G_{t} G_{r} G M^{2} N^{2} d_{x} d_{y} \lambda^{2} F\left(\theta_{t}, \varphi_{t}\right) F\left(\theta_{r}, \varphi_{r}\right) A^{2}}{64 \pi^{3} d_{1}{ }^{2} d_{2}{ }^{2}} \\
& \times \mid \frac{\operatorname{sinc}\left(\frac{M \pi}{\lambda}\left(\sin \theta_{t} \cos \varphi_{t}+\sin \theta_{r} \cos \varphi_{r}+\delta_{1}\right) d_{x}\right)}{\operatorname{sinc}\left(\frac{\pi}{\lambda}\left(\sin \theta_{t} \cos \varphi_{t}+\sin \theta_{r} \cos \varphi_{r}+\delta_{1}\right) d_{x}\right)} \\
& \times\left.\frac{\operatorname{sinc}\left(\frac{N \pi}{\lambda}\left(\sin \theta_{t} \sin \varphi_{t}+\sin \theta_{r} \sin \varphi_{r}+\delta_{2}\right) d_{y}\right)}{\operatorname{sinc}\left(\frac{\pi}{\lambda}\left(\sin \theta_{t} \sin \varphi_{t}+\sin \theta_{r} \sin \varphi_{r}+\delta_{2}\right) d_{y}\right)}\right|^{2},
\end{aligned}
$$

where $\delta_{1}\left(m-\frac{1}{2}\right) d_{x}+\delta_{2}\left(n-\frac{1}{2}\right) d_{y}=\frac{\lambda \phi_{n, m}}{2 \pi}$. When $\theta_{r}=\theta_{\text {des }}$ and $\varphi_{r}=\varphi_{\text {des }}$, (7) is maximized as

$$
P_{r}^{\max }=P_{t} \frac{G_{t} G_{r} G M^{2} N^{2} d_{x} d_{y} \lambda^{2} F\left(\theta_{t}, \varphi_{t}\right) F\left(\theta_{d e s}, \varphi_{d e s}\right) A^{2}}{64 \pi^{3} d_{1}{ }^{2} d_{2}{ }^{2}}
$$

\footnotetext{
${ }^{5}$ The intelligent reflection in this paper refers to intelligently controlling the reflection nature of the RIS by properly programming the reflection coefficients of the unit cells of the RIS. The specular reflection in this paper includes two cases: far-field specular beamforming and near-field specular broadcasting, and the reflection coefficients of the unit cells are identical.
}

when $\delta_{1}=-\sin \theta_{t} \cos \varphi_{t}-\sin \theta_{\text {des }} \cos \varphi_{\text {des }}$ and $\delta_{2}=$ $-\sin \theta_{t} \sin \varphi_{t}-\sin \theta_{\text {des }} \sin \varphi_{\text {des }}$. $\phi_{n, m}$ is assumed to be continuous for free-space path loss modeling in this paper [12] [13], and the corresponding design of $\phi_{n, m}$ is

$$
\begin{gathered}
\phi_{n, m}=\bmod \left(\frac{2 \pi}{\lambda}\left(\delta_{1}\left(m-\frac{1}{2}\right) d_{x}+\delta_{2}\left(n-\frac{1}{2}\right) d_{y}\right), 2 \pi\right) \\
=\bmod \left(-\frac{2 \pi}{\lambda}\left(\left(\sin \theta_{t} \cos \varphi_{t}+\sin \theta_{\text {des }} \cos \varphi_{\text {des }}\right)\left(m-\frac{1}{2}\right) d_{x}\right.\right. \\
\left.\left.\quad+\left(\sin \theta_{t} \sin \varphi_{t}+\sin \theta_{\text {des }} \sin \varphi_{\text {des }}\right)\left(n-\frac{1}{2}\right) d_{y}\right), 2 \pi\right) .
\end{gathered}
$$

Proof: See Appendix C.

According to the phase shift design described by (9), the incident signal from direction $\left(\theta_{t}, \varphi_{t}\right)$ to the RIS can be manipulated and reflected towards any desired direction $\left(\theta_{\text {des }}, \varphi_{\text {des }}\right)$ in the far field case, which is referred to as intelligent reflection. Besides, (4) and (7) also indicate that the free-space path loss of RIS-assisted far-field beamforming has the same scaling law as for specular reflection and intelligent reflection. Moreover, as for the far-field beamforming case, a large-size RIS outperforms a small-size RIS under the same condition based on (6).

\section{Near Field Beamforming Case}

In the previous subsection, the path loss analyses for the RIS-assisted beamforming assume that both of the transmitter and the receiver are in the far field of the RIS. The case study where the transmitter and/or the receiver are in the near field of the RIS is discussed in this subsection, which enables the RIS to focus the reflected signal to the receiver by properly design the reflection coefficients $\Gamma_{n, m}$.

Proposition 3: Assume that the reflection coefficients of all the unit cells share the same amplitude value $A$ and different phase shifts $\phi_{n, m}$ and that the RIS-assisted wireless communication system operates in the near field case. The received signal power is written as follows

$$
\begin{aligned}
& P_{r}=P_{t} \frac{G_{t} G_{r} G d_{x} d_{y} \lambda^{2} A^{2}}{64 \pi^{3}} \\
& \times\left|\sum_{m=1-\frac{M}{2}}^{\frac{M}{2}} \sum_{n=1-\frac{N}{2}}^{\frac{N}{2}} \sqrt{F_{n, m}^{\text {combine }}} \frac{e^{\frac{-j\left(2 \pi\left(r_{n, m}^{t}+r_{n, m}^{r}\right)-\lambda \phi_{n, m}\right)}{\lambda}}}{r_{n, m}^{t} r_{n, m}^{r}}\right|^{2} .
\end{aligned}
$$




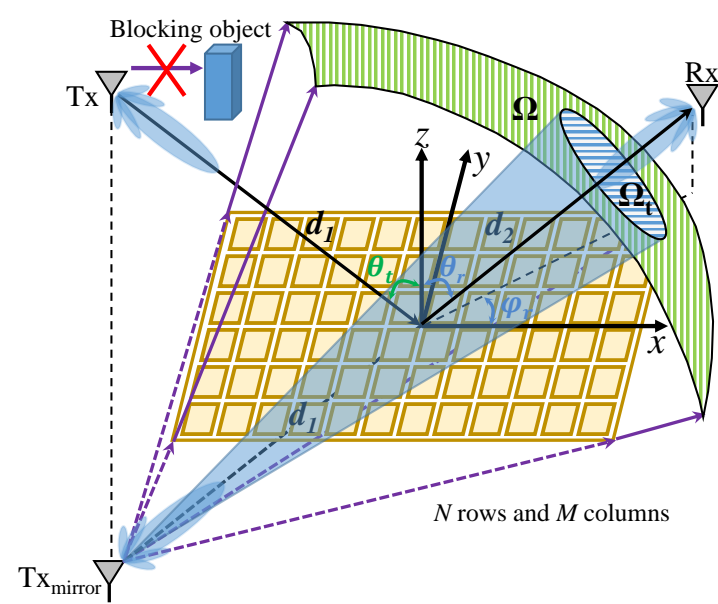

(a)

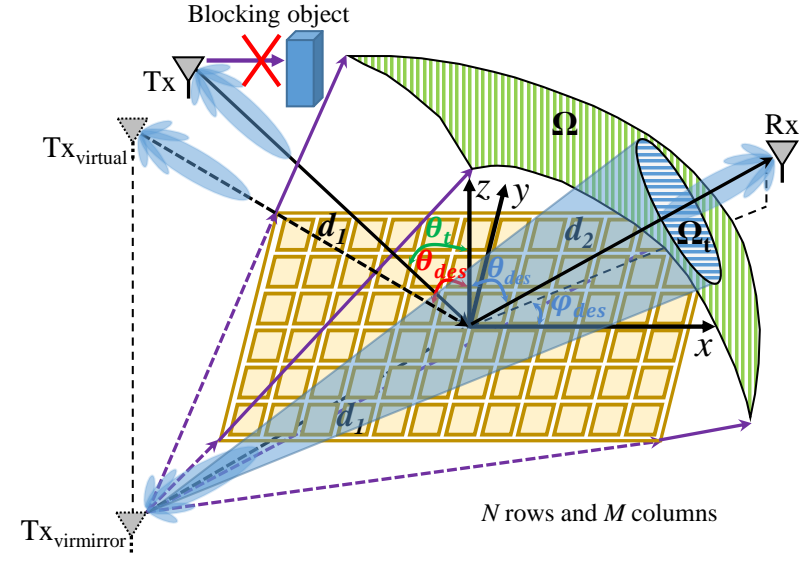

(b)

Fig. 4. Near field broadcasting case of RIS-assisted wireless communication in the view of geometrical optics. (a) Broadcasting through specular reflection. (b) Broadcasting through intelligent reflection.

For the desired receiver at any position $\left(x_{r}, y_{r}, z_{r}\right), 10$ is maximized as

$$
P_{r}^{\max }=P_{t} \frac{G_{t} G_{r} G d_{x} d_{y} \lambda^{2} A^{2}}{64 \pi^{3}}\left|\sum_{m=1-\frac{M}{2}}^{\frac{M}{2}} \sum_{n=1-\frac{N}{2}}^{\frac{N}{2}} \frac{\sqrt{F_{n, m}^{\text {combine }}}}{r_{n, m}^{t} r_{n, m}^{r}}\right|^{2},
$$

when

$$
\phi_{n, m}=\bmod \left(\frac{2 \pi\left(r_{n, m}^{t}+r_{n, m}^{r}\right)}{\lambda}, 2 \pi\right) .
$$

The path loss corresponding to (11) is

$$
P L_{\text {nearfield }}^{\text {beam }}=\frac{64 \pi^{3}}{G_{t} G_{r} G d_{x} d_{y} \lambda^{2} A^{2}\left|\sum_{m=1-\frac{M}{2}}^{\frac{M}{2}} \sum_{n=1-\frac{N}{2}}^{\frac{N}{2}} \frac{\sqrt{F_{n, m}^{c o m b i n e}}}{r_{n, m}^{t} r_{n, m}^{r}}\right|^{2}} .
$$

Proof: See Appendix D.

Proposition 3 gives the phase gradient design of the RIS for the maximization of the received signal power in the near field case and equation (11) is referred to as the near-field beamforming formula. Proposition 3 also formulates the freespace path loss of RIS-assisted near field beamforming case. However, its dependence on $d_{1}$ and $d_{2}$ cannot be directly inferred from (13), which will be further discussed based on the simulation results in the next section.

\section{Near Field Broadcasting Case}

The studies conducted in the previous subsections assume that the RIS is employed for beamforming, and, then, the received power is maximized for one specific user. The scenario where the RIS is utilized for broadcasting is discussed in this subsection. When the transmitter is in the near field of the RIS, it means that the transmitter is relatively near to the RIS, thus the electromagnetic wave transmitted to the RIS can be regarded as spherical wave as discussed in Section II. If the RIS is electrically large (i.e., both of its length $M d_{x}$ and width $N d_{y}$ are at least 10 times larger than the wavelength $\lambda$ ), then the incident spherical wave will form a circular and divergent phase gradient on the RIS surface, which is due to the different transmission distances from the transmitter to each unit cell of the RIS.

Proposition 4: Assume that all the unit cells share the same reflection coefficient $\Gamma_{n, m}=A e^{j \phi}$, the received signal power through the reflection of an RIS of large electrical size in the near field broadcasting case can be approximately formulated as follows

$P_{r} \approx\left\{\begin{array}{rr}\frac{G_{t} A_{r} A^{2}}{4 \pi\left(d_{1}+d_{2}\right)^{2}} P_{t}=\frac{G_{t} G_{r} \lambda^{2} A^{2}}{16 \pi^{2}\left(d_{1}+d_{2}\right)^{2}} P_{t}, & \left(\theta_{r}, \varphi_{r}\right) \in \Omega \cap \Omega_{t} \\ 0, & \left(\theta_{r}, \varphi_{r}\right) \notin \Omega \cap \Omega_{t}\end{array}\right.$

where $A_{r}=\frac{G_{r} \lambda^{2}}{4 \pi}$, and the solid angle $\Omega$ is enclosed by the extension lines from the mirror image of the transmitter $\left(\mathrm{Tx}_{\text {mirror }}\right)$ to the edge of the RIS, as illustrated in Fig. 4(a). The solid angle $\Omega_{t}$ is formed by the main lobe of the transmitting antenna of $\mathrm{Tx}_{\text {mirror }}$. If the elevation angle $\theta_{r}$ and the azimuth angle $\varphi_{r}$ of the receiver are within the intersection of $\Omega$ and $\Omega_{t}$, the receiver can receive the reflected signal from the RIS, otherwise it can hardly receive any signal. The RIS performs specular reflection in this near-field broadcasting case, and its free-space path loss is

$$
P L_{\text {nearfield }}^{\text {broadcast }} \approx \frac{16 \pi^{2}\left(d_{1}+d_{2}\right)^{2}}{G_{t} G_{r} \lambda^{2} A^{2}} .
$$

Proof: In the near field broadcasting case, the free-space path loss of RIS-assisted wireless communications can also be characterized by the general formula $(3)$ in Theorem 1. In addition, a more insightful result can be obtained by using the approximation based on geometric optics, which is typically employed in ray tracing. Since all the unit cells share the same reflection coefficient, the RIS performs specular reflection in the view of geometrical optics. As shown in Fig. 4(a), the mirror image of the transmitter, namely $\mathrm{Tx}_{\text {mirror }}$, can be obtained by taking the RIS as the symmetric plane. The signal transmission process is equivalent to that the signal is transmitted from $\mathrm{Tx}_{\text {mirror }}$ and received by the receiver after travelling distance $\left(d_{1}+d_{2}\right)$, thus we can get Proposition 4 according to the conventional free-space path loss model [42].

The above analysis assumes that all the unit cells share the same reflection coefficient. In the following, we introduce a 
TABLE I

Free-Space Path Loss Models of RiS-ASsisted Wireless Communications in Different Scenarios.

\begin{tabular}{|c|c|c|c|}
\hline Applications & Near/Far Field Conditions & Path Loss & Notes \\
\hline Beamforming & $\begin{array}{l}\text { Transmitter and receiver are both in the } \\
\text { far field of RIS }\end{array}$ & 6 & $\begin{array}{l}\text { Apply to both electrically small and large RISs } \\
\phi_{n, m} \text { are designed as in } 9\end{array}$ \\
\hline Beamforming & $\begin{array}{l}\text { Transmitter and receiver are both or only } \\
\text { one of them is in the near field of RIS }\end{array}$ & 13) & $\begin{array}{l}\text { Apply to both electrically small and large RISs } \\
\phi_{n, m} \text { are designed as in } 12\end{array}$ \\
\hline Broadcasting & $\begin{array}{l}\text { Transmitter and receiver are both or only } \\
\text { one of them is in the near field of RIS }\end{array}$ & 15 & $\begin{array}{l}\text { Only apply to electrically large RIS } \\
\phi_{n, m} \text { are designed as in } 16\end{array}$ \\
\hline
\end{tabular}

TABLE II

The Parameters of Three Different RISs and Two Different Antennas Utilized in Simulations.

\begin{tabular}{|c|c|}
\hline Name & Parameters \\
\hline Large RIS1 & $\begin{array}{l}N=100, M=102, d_{x}=d_{y}=0.01 \mathrm{~m},\left|\Gamma_{n, m}\right|=A=0.9, F(\theta, \varphi) \text { is defined by (1), } \\
\text { operating frequency } f=10.5 \mathrm{GHz}, \lambda=c / f=0.0286 \mathrm{~m}, \frac{2 D^{2}}{\lambda}=\frac{2 M N d_{x} d_{y}}{\lambda}=71.4 \mathrm{~m} .\end{array}$ \\
\hline Large RIS2 & $\begin{array}{l}N=50, M=34, d_{x}=d_{y}=0.01 \mathrm{~m},\left|\Gamma_{n, m}\right|=A=0.9, F(\theta, \varphi) \text { is defined by } 11 \\
\text { operating frequency } f=10.5 \mathrm{GHz}, \lambda=c / f=0.0286 \mathrm{~m}, \frac{2 D^{2}}{\lambda}=\frac{2 M N d_{x} d_{y}}{\lambda}=11.9 \mathrm{~m} .\end{array}$ \\
\hline Small RIS & $\begin{array}{l}\left.N=8, M=32, d_{x}=d_{y}=0.012 m,\left|\Gamma_{n, m}\right|=A=0.7, F(\theta, \varphi) \text { is defined by } 1\right] \\
\text { operating frequency } f=4.25 \mathrm{GHz}, \lambda=c / f=0.07 \mathrm{~m}, \frac{2 D^{2}}{\lambda}=\frac{2 M N d_{x} d_{y}}{\lambda}=1 \mathrm{~m} .\end{array}$ \\
\hline $\mathrm{X}$-band horn antenna & $\begin{array}{l}F^{t x}(\theta, \varphi)=F^{r x}(\theta, \varphi)=\cos ^{62} \theta \text { when } \theta \in\left[0, \frac{\pi}{2}\right], F^{t x}(\theta, \varphi)=F^{r x}(\theta, \varphi)=0 \text { when } \theta \in\left(\frac{\pi}{2}, \pi\right] . \\
\left.G_{t}=G_{r}=21 d B \text { according to } 2\right) \text { under the assumption that the radiation efficiency is } 100 \% . \\
\text { The operating frequency is } f=10.5 \mathrm{GHz} \text {. }\end{array}$ \\
\hline C-band horn antenna & $\begin{array}{l}F^{t x}(\theta, \varphi)=F^{r x}(\theta, \varphi)=\cos ^{13} \theta \text { when } \theta \in\left[0, \frac{\pi}{2}\right], F^{t x}(\theta, \varphi)=F^{r x}(\theta, \varphi)=0 \text { when } \theta \in\left(\frac{\pi}{2}, \pi\right] . \\
G_{t}=G_{r}=14.5 d B \text { according to } \sqrt{2} \text { under the assumption that the radiation efficiency is } 100 \% . \\
\text { The operating frequency is } f=4.25 \mathrm{GHz} \text {. }\end{array}$ \\
\hline
\end{tabular}

design method for the phase shifts of the unit cells, which enables the RIS to broadcast the reflected signal to the desired direction through intelligent reflection.

Proposition 5: Assume that the reflection coefficients of all the unit cells share the same amplitude value $A$ and different phase shift $\phi_{n, m}$, and the RIS aims to broadcast the reflected signal to the desired direction $\left(\theta_{\text {des }}, \varphi_{\text {des }}\right)$ (i.e., the desired center direction of $\Omega_{t}$ ) in the near field broadcasting case, an effective design method for the phase shift $\phi_{n, m}$ is the following

$$
\phi_{n, m}=\bmod \left(\frac{2 \pi}{\lambda}\left(r_{n, m}^{t}-r_{n, m}^{v i r t}\right), 2 \pi\right)
$$

where $r_{n, m}^{t}$ represents the distance between the transmitter and the unit cell $U_{n, m}, r_{n, m}^{\text {virt }}$ represents the distance between the virtual transmitter $T x_{\text {virtual }}$ and the unit cell $U_{n, m}$. The position of the virtual transmitter is $\left(-d_{1} \sin \theta_{\text {des }} \cos \varphi_{\text {des }},-d_{1} \sin \theta_{\text {des }} \sin \varphi_{\text {des }}, d_{1} \cos \theta_{\text {des }}\right)$ as shown in Fig. 4(b).

Proof: If all the unit cells of the RIS share the same phase shift, i.e., if the RIS is a equiphase surface, it will perform specular reflection in the near-field broadcasting case according to geometric optics. Therefore, as shown in Fig. 4(b), if a virtual transmitter $T x_{\text {virtual }}$ is placed in the mirror direction $\left(\theta_{\text {des }}, \varphi_{\text {des }}+\pi\right)$ of the desired direction $\left(\theta_{\text {des }}, \varphi_{\text {des }}\right)$ and its distance to the center of RIS is $d_{1}$, the RIS will broadcast the reflected signal to the desired direction $\left(\theta_{d e s}, \varphi_{\text {des }}\right)$. Since the real transmitter $T x$ usually doesn't coincide with the imagined virtual transmitter in position, phase compensation can be used to make the real transmitter to behave as the virtual transmitter. As $-\frac{2 \pi}{\lambda} r_{n, m}^{t}$ and $-\frac{2 \pi}{\lambda} r_{n, m}^{v i r t}$ are the phase alterations caused by free-space propagation from the real transmitter and the virtual transmitter to the unit cell $U_{n, m}$, respectively, the phase shifts of the unit cells can be designed as in 16 to compensate for the phase difference between them. With this design, the reflected signal is broadcasted to the desired direction. In addition, the signal transmission process is equivalent to that of a signal is transmitted from $T x_{\text {virmirror }}$ (the mirror image of the virtual transmitter) and received by the receiver after travelling distance $\left(d_{1}+d_{2}\right)$ as shown in Fig. 4(b), thus (15) also holds for the intelligent near field broadcasting.

Propositions 4 and 5 indicate that when the transmitter is in the near field of an RIS with large electrical size and the phase shifts $\phi_{n, m}$ are designed as in $(16)$, the free-space path loss of RIS-assisted wireless communications is proportional to $\left(d_{1}+d_{2}\right)^{2}$, instead of $d_{1}{ }^{2} d_{2}{ }^{2}$ as for the far-field case. Similarly, when the receiver is in the near field of the RIS, the same result holds according to the transmitter-receiver reciprocity property revealed by Theorem 1. Formula 14 is referred to as the near-field broadcasting formula, which describes the relationship between the received and transmitted signal power of RIS-assisted wireless communications in the near field broadcasting case, and is more insightful compared with the general formula 3 in Theorem 1.

\section{E. Path Loss Model Summary}

Based on the modeling results above, the free-space path loss models of RIS-assisted wireless communications in different scenarios 6 are summarized in Table I

\footnotetext{
${ }^{6}$ The far field broadcasting case is not discussed in this paper, mainly because it is not that suitable for wireless communications. In the far-field case, the path loss of every individual link between the transmitter and receiver provided by each unit cell of the RIS is very large $\left(\propto\left(d_{1} d_{2}\right)^{2}\right)$, so it is necessary to perform beamforming to guarantee the quality of RISassisted link, rather than broadcasting. In fact, the "far field broadcasting" case is usually used in the research field of metasurface-based electromagnetic diffusion [43] [44], which is suitable for the application of radar cross-section (RCS) reduction.
} 


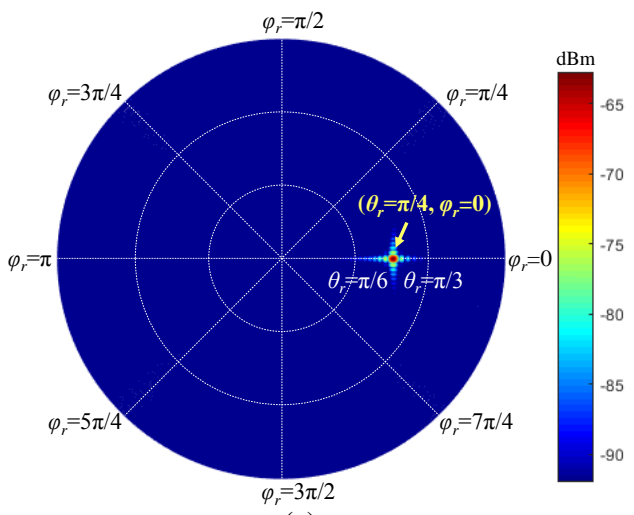

(a)

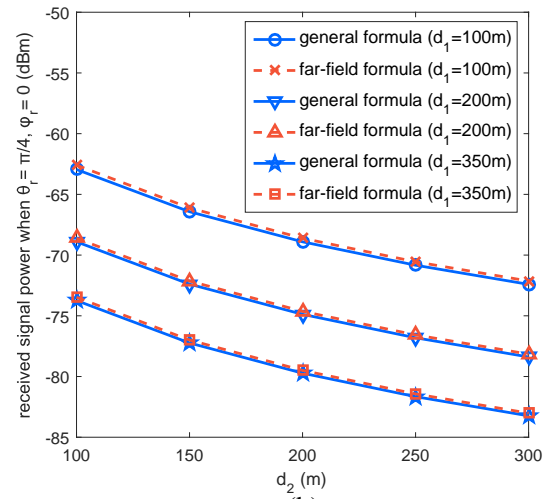

(b)

Fig. 5. Simulation results of RIS-assisted beamforming through specular reflection of large RIS1 in the far field case. (a) Received signal power distribution when $d_{1}=d_{2}=100 \mathrm{~m}$. (b) Received signal power in the specular direction versus $d_{1}$ and $d_{2}$.

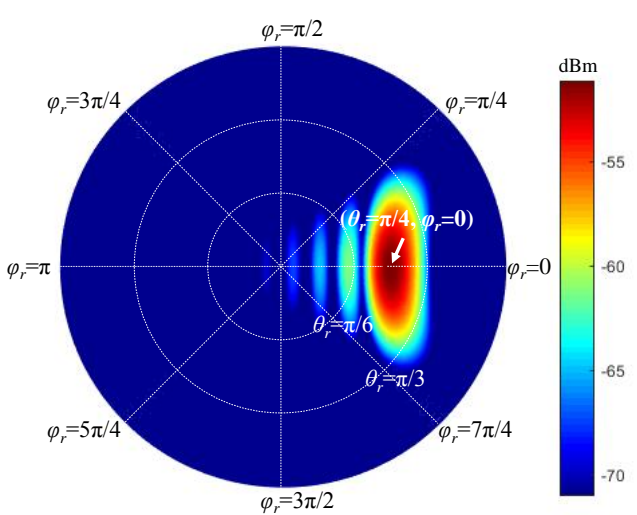

(a)

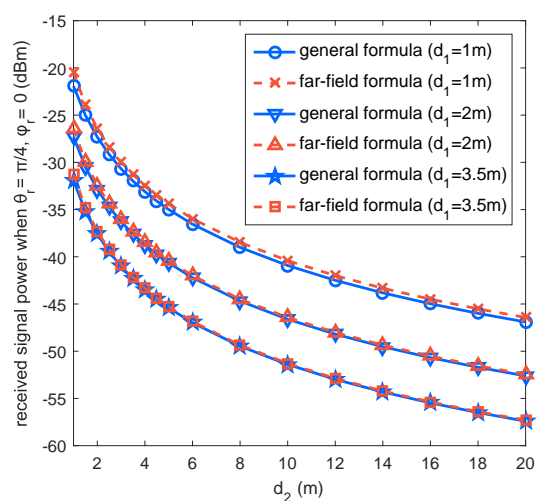

(b)

Fig. 6. Simulation results of RIS-assisted beamforming through specular reflection of small RIS in the far field case. (a) Received signal power distribution when $d_{1}=3.5 \mathrm{~m}$ and $d_{2}=10 \mathrm{~m}$. (b) Received signal power in the specular direction versus $d_{1}$ and $d_{2}$.

\section{VAlidation of Path Loss Models Via Numerical Simulations}

Numerical simulations are conducted based on the general formula (3) to verify the far-field formula (4), the near-field beamforming formula (11), the near-field broadcasting formula (14), and their corresponding path loss formulas (6), (13) and (15), respectively. Three different RISs and two different antennas (corresponding to those used in our measurements, described in Section V) are utilized in the simulations, with their parameters specified in Table II The electrical sizes of the RISs are $35 \lambda \times 35.7 \lambda$ for the large RIS1, $17.5 \lambda \times 11.9 \lambda$ for the large RIS2, and $1.36 \lambda \times 5.44 \lambda$ for the small RIS, respectively.

\section{A. RIS-assisted Beamforming}

1) Specular Reflection in the Far Field Beamforming Case: $P_{t}=0 \mathrm{dBm}, \theta_{t}=\frac{\pi}{4}, \varphi_{t}=\pi, \angle \Gamma_{n, m}=\phi_{n, m}=0$. The large RIS1 and X-band antennas are employed in the simulation. When $d_{1}$ and $d_{2}$ are both greater than $71.4 \mathrm{~m}$, the far field condition of RIS1 is fulfilled. The small RIS and C-band antennas are also employed in the simulation under the same conditions. When $d_{1}$ and $d_{2}$ are both larger than $1 \mathrm{~m}$, the far field condition of the small RIS is fulfilled. Fig. 5(a) and Fig. 6(a) show the simulated received signal power distribution in various directions in the large RIS1 case and the small RIS case, respectively. It can be seen that the received signal power is maximized when $\theta_{r}=\frac{\pi}{4}$ and $\varphi_{r}=0$, which indicates that both of the large RIS1 and the small RIS specularly reflect the incident signal. Moreover, the larger the electrical size of the RIS is, the more concentrated the power of the reflected signal. As shown in Fig. 5.b) and Fig. 6(b), the far-field formula (4) matches well with the general formula (3), which validates that the free-space path loss is compliant with (6) under the scenario of RIS-assisted beamforming through specular reflection of RIS in the far field case.

2) Intelligent Reflection in the Far Field Beamforming Case: $P_{t}=0 \mathrm{dBm}, \theta_{t}=\frac{\pi}{4}, \varphi_{t}=\pi$. The large RIS1 and X-band antennas are employed in the simulation, and the phase shifts $\phi_{n, m}$ are designed to steer the reflected signal to a desired direction of $\theta_{\text {des }}=\frac{\pi}{3}$ and $\varphi_{\text {des }}=\frac{7 \pi}{4}$ according to (9). The small RIS and C-band antennas are also employed in the simulation, and $\phi_{n, m}$ are designed to steer the reflected signal to a desired direction of $\theta_{\text {des }}=\frac{\pi}{6}$ and $\varphi_{\text {des }}=0$. Fig. 7 (a) and Fig. 8(a) show that the received signal power is maximized when $\theta_{r}=\frac{\pi}{3}, \varphi_{r}=\frac{7 \pi}{4}$ and $\theta_{r}=\frac{\pi}{6}, \varphi_{r}=0$, respectively, which indicates that both of the large RIS1 and the small RIS successfully reflect the incident signal towards the desired direction. As shown in Fig. 7(b) and Fig. 8(b), the far-field formula (7) with phase design matches well with the general formula (3), which validates that the free-space 


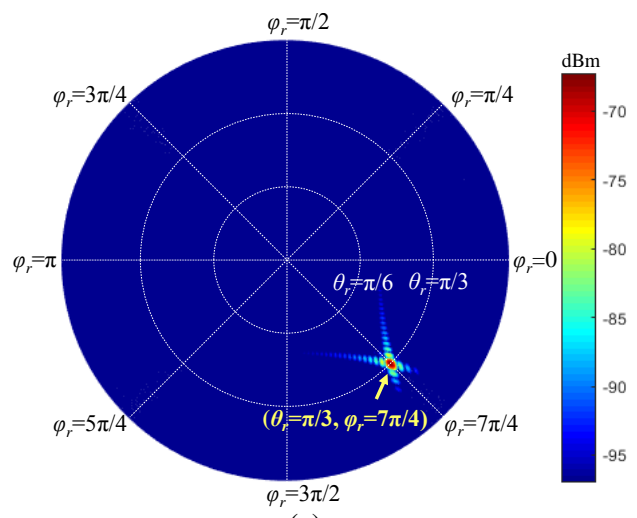

(a)

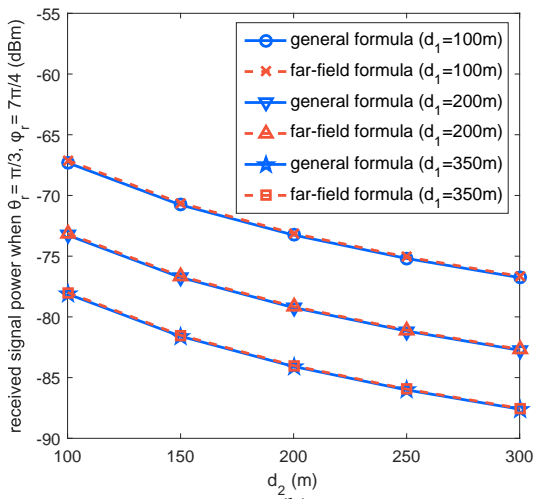

(b)

Fig. 7. Simulation results of RIS-assisted beamforming through intelligent reflection of large RIS1 in the far field case. (a) Received signal power distribution case when $\theta_{\text {des }}=\frac{\pi}{3}, \varphi_{\text {des }}=\frac{7 \pi}{4}, d_{1}=100 \mathrm{~m}, d_{2}=100 \mathrm{~m}$. (b) Received signal power in the desired direction versus $d_{1}$ and $d_{2}$.

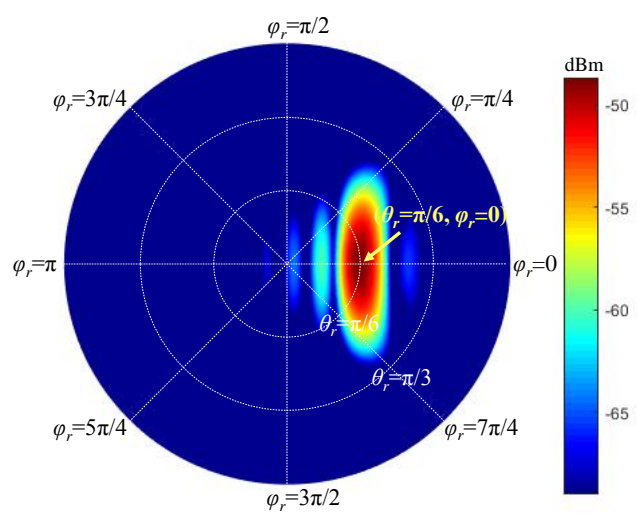

(a)

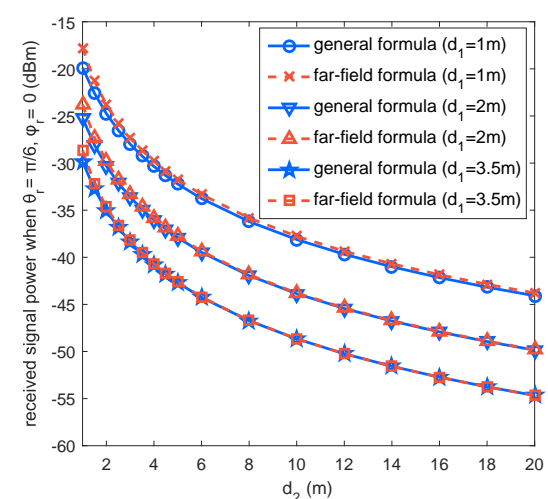

(b)

Fig. 8. Simulation results of RIS-assisted beamforming through intelligent reflection of small RIS in the far field case. (a) Received signal power distribution when $\theta_{\text {des }}=\frac{\pi}{6}, \varphi_{\text {des }}=0, d_{1}=3.5 \mathrm{~m}, d_{2}=10 \mathrm{~m}$. (b) Received signal power in the desired direction versus $d_{1}$ and $d_{2}$.

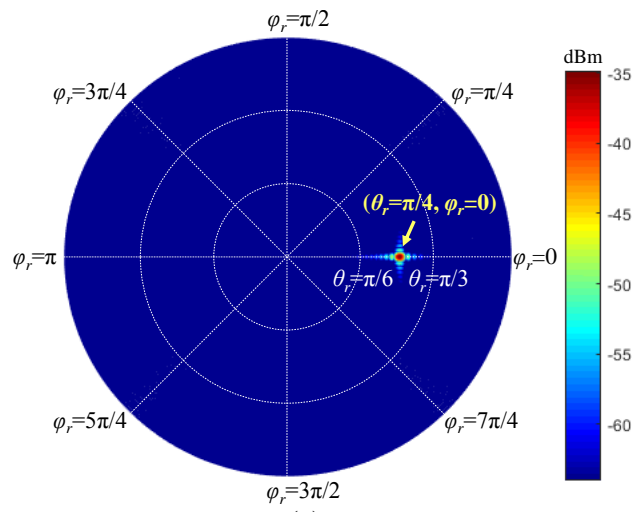

(a)

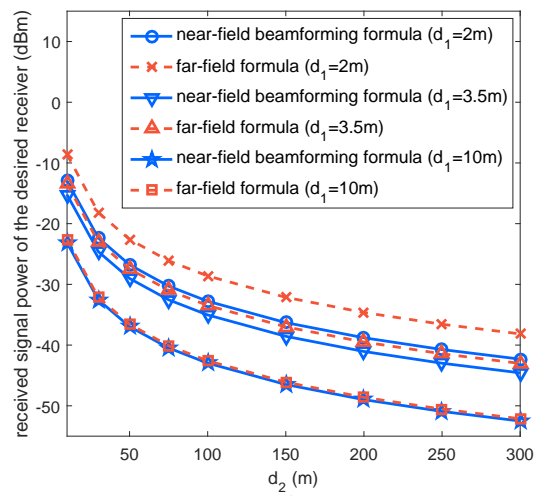

(b)

Fig. 9. Simulation results of RIS-assisted beamforming through intelligent reflection of large RIS1 in the near field case. (a) Received signal power distribution when $d_{1}=3.5 \mathrm{~m}$ and the desired receiver is at $d_{2}=100 \mathrm{~m}, \theta_{r}=\frac{\pi}{4}$ and $\varphi_{r}=0$. (b) Received signal power of the desired receiver versus $d_{1}$ and $d_{2}$. 


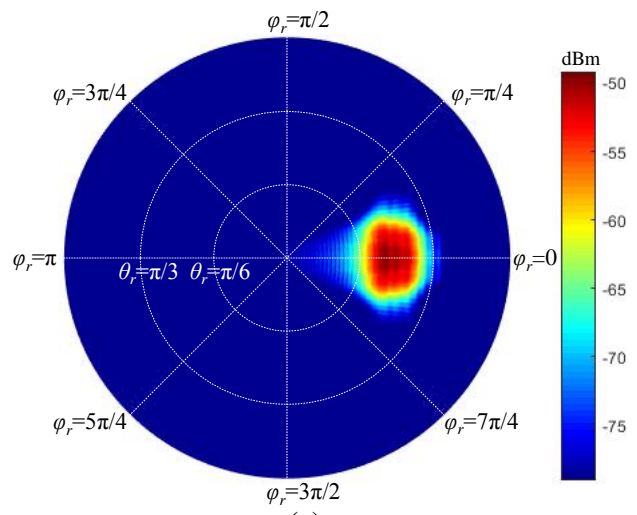

(a)

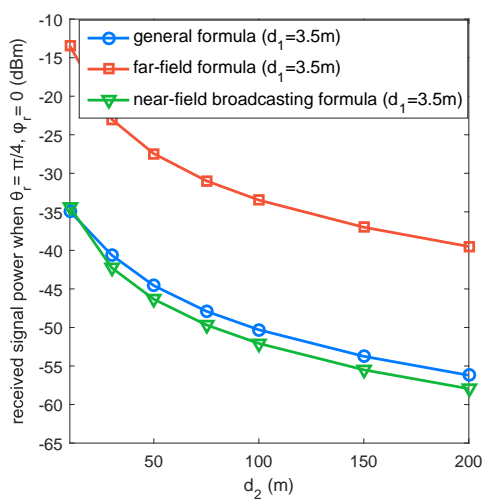

(c)

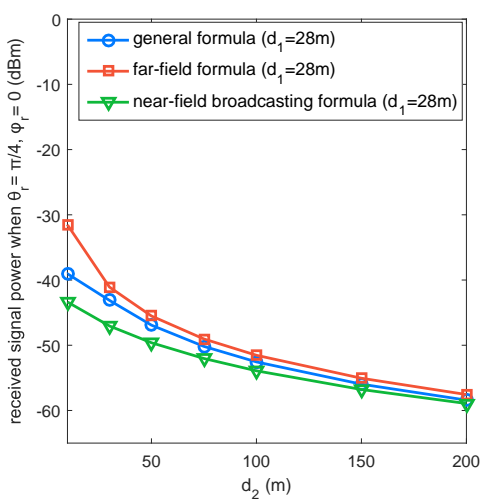

(d)

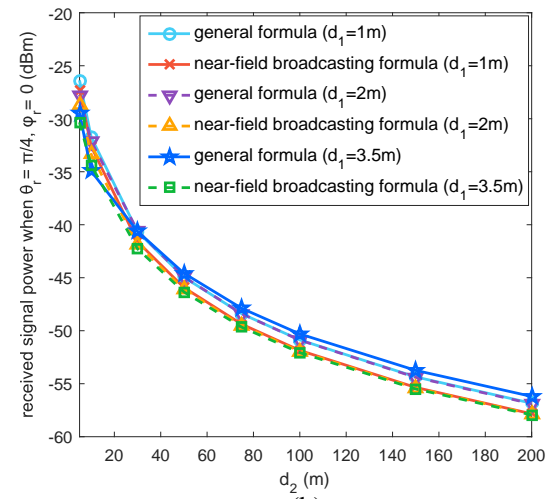

(b)

Fig. 10. Simulation results of RIS-assisted broadcasting through specular reflection of large RIS1 in the near field case. (a) Received signal power distribution when $d_{1}=2 \mathrm{~m}$ and $d_{2}=100 \mathrm{~m}$. (b) Received signal power of the broadcasted receiver versus $d_{1}$ and $d_{2}$ when $d_{1}=1 \mathrm{~m}, 2 \mathrm{~m}$, and $3.5 \mathrm{~m}$. (c)-(e) Received signal power of the broadcasted receiver versus $d_{1}$ and $d_{2}$ when $d_{1}=3.5 \mathrm{~m}, 28 \mathrm{~m}$, and $50 \mathrm{~m}$, respectively.

path loss adheres to (6) under the scenario of RIS-assisted beamforming through intelligent reflection of RIS in the far field case.

3) Intelligent Reflection in the Near Field Beamforming Case: $P_{t}=0 \mathrm{dBm}, \theta_{t}=\frac{\pi}{4}, \varphi_{t}=\pi$. The large RIS1 and $\mathrm{X}$-band antennas are employed in the simulation, and $\phi_{n, m}$ are designed to focus the reflected signal towards the desired receiver at a certain location of $d_{2}=100 \mathrm{~m}, \theta_{r}=\frac{\pi}{4}$ and $\varphi_{r}=0$ according to 12 . The transmitter is in the near field of RIS1. Fig. 9 (a) shows the power distribution of the simulated received signal when $d_{1}=3.5 \mathrm{~m}$ and $d_{2}=100 \mathrm{~m}$. It can be observed that the reflected signal has been successfully focused towards the desired receiver $\left(d_{2}=100 \mathrm{~m}, \theta_{r}=\frac{\pi}{4}\right.$ and $\varphi_{r}=0$ ) by applying (12). Fig. 9 (b) shows that as $d_{1}$ increases, the near-field beamforming formula (11) fits better with the far-field formula (4), although the transmitter is in the near field of the RIS. This is because, when both $d_{1}$ and $d_{2}$ increase, the distances between the transmitter/receiver and each unit cell become more similar, and the RIS is gradually covered by the main lobe of the transmitting/receiving antenna. Therefore, 111 can be written as

$$
P_{r}^{\max } \approx P_{t} \frac{G_{t} G_{r} G d_{x} d_{y} \lambda^{2} A^{2}}{64 \pi^{3}} \frac{M^{2} N^{2} F\left(\theta_{t}, \varphi_{t}\right) F\left(\theta_{r}, \varphi_{r}\right)}{d_{1}^{2} d_{2}^{2}}
$$

by applying $F^{t x}\left(\theta_{n, m}^{t x}, \varphi_{n, m}^{t x}\right) \approx 1, F^{r x}\left(\theta_{n, m}^{r x}, \varphi_{n, m}^{r x}\right) \approx 1$, $F\left(\theta_{n, m}^{t}, \varphi_{n, m}^{t}\right) \approx F\left(\theta_{\mathrm{t}}, \varphi_{t}\right), F\left(\theta_{n, m}^{r}, \varphi_{n, m}^{r}\right) \approx F\left(\theta_{r}, \varphi_{r}\right)$, $r_{n, m}^{t} \approx d_{1}$, and $r_{n, m}^{r} \approx d_{2}$ into $(11)$. Formula $\sqrt{17}$ is exactly the same as (5), which reveals that the free-space path loss gradually approaches (6) under the RIS-assisted beamforming scenario in the near field case when both $d_{1}$ and $d_{2}$ increase. The above conclusion also holds for the small RIS as it is not related to the size of RIS.

\section{B. RIS-assisted Broadcasting}

1) Specular Reflection in the Near Field Broadcasting Case: $P_{t}=0 \mathrm{dBm}, \theta_{t}=\frac{\pi}{4}, \varphi_{t}=\pi, \angle \Gamma_{n, m}=\phi_{n, m}=0$. The large RIS1 and X-band antennas are employed in the simulation. When $d_{1}$ or $d_{2}$ is less than $71.4 \mathrm{~m}$, the near field condition of the large RIS1 is fulfilled. Fig. 10.a) shows the simulated received signal power distribution in various directions when $d_{1}=2 \mathrm{~m}$ and $d_{2}=100 \mathrm{~m}$. It can be seen that a specific area which is defined as the solid angle $\Omega \cap \Omega_{t}$ in $(14$ is lit up. As shown in Fig. 10 b), the near-field broadcasting formula (14) fits well with the general formula (3) when $d_{1}=1$ $\mathrm{m}, 2 \mathrm{~m}$, and $3.5 \mathrm{~m}$, which reveals that the free-space path loss follows (15) under the scenario of RIS-assisted broadcasting through specular reflection of a large RIS in the near field case. In addition, the general formula curve gradually moves from the near-field broadcasting formula curve to the far-field formula curve as $d_{1}$ increases as shown in Fig. 10.c)-(e), which indicates a transition process between the near field and the far field of the RIS. It is worth noting that the curves of the farfield formula and the near-field broadcasting formula almost overlap when $d_{1}=28 \mathrm{~m}$. Therefore, we redefine the boundary 


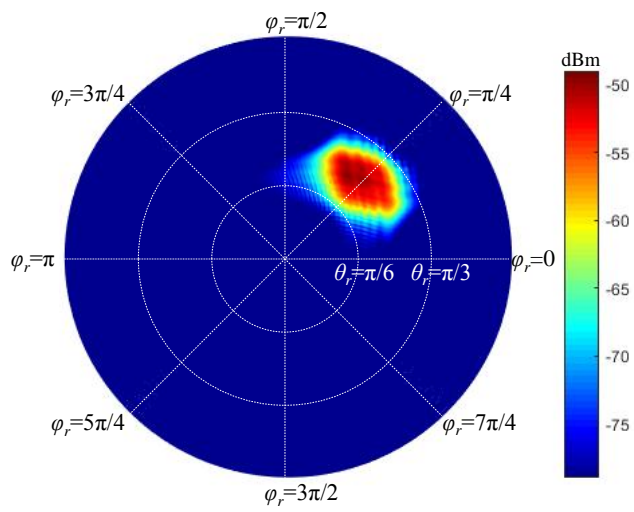

(a)

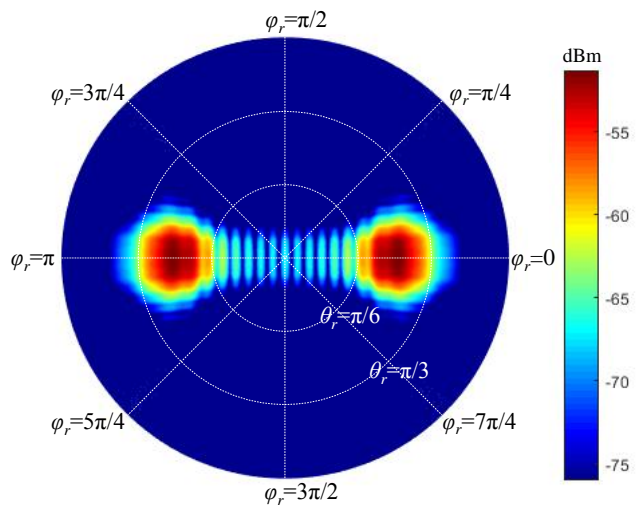

(c)

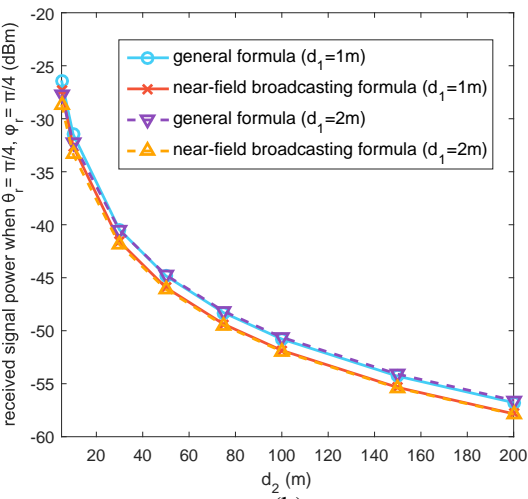

(b)

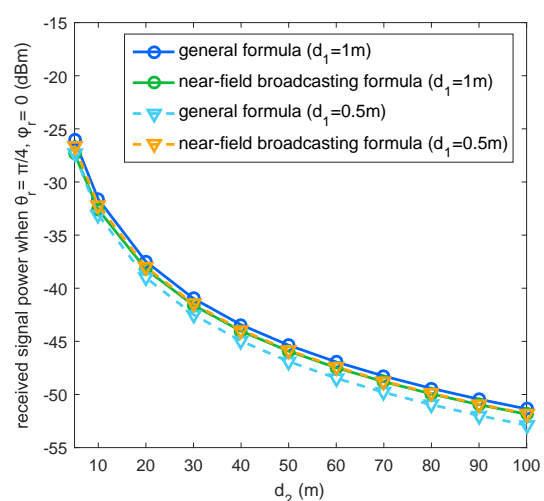

(d)

Fig. 11. Simulation results of RIS-assisted near-field broadcasting through intelligent reflection. (a) Received signal power distribution when $d_{1}=2 \mathrm{~m}$ and $d_{2}=100 \mathrm{~m}$ with large RIS1. (b) Received signal power versus $d_{1}$ and $d_{2}$ when $d_{1}=1 \mathrm{~m}$ and $2 \mathrm{~m}$ with large RIS1. (c) Received signal power distribution when $d_{1}=1 \mathrm{~m}$ and $d_{2}=100 \mathrm{~m}$ with large RIS2. (d) Received signal power versus $d_{1}$ and $d_{2}$ when $d_{1}=0.5 \mathrm{~m}$ and $1 \mathrm{~m}$ with large RIS2.

of the far field and the near field of the RIS as $L_{\text {bound }}$, by letting (6) equals to (15) as

$$
\begin{aligned}
& \frac{G_{t} G_{r} G M^{2} N^{2} d_{x} d_{y} \lambda^{2} F\left(\theta_{t}, \varphi_{t}\right) F\left(\theta_{r}, \varphi_{r}\right) A^{2}}{64 \pi^{3} L_{\text {bound }}^{2} d_{2}^{2}} \\
& =\frac{G_{t} G_{r} \lambda^{2} A^{2}}{16 \pi^{2}\left(L_{\text {bound }}+d_{2}\right)^{2}} .
\end{aligned}
$$

When $d_{2}$ is much larger than $L_{\text {bound }}$ (the receiver is in the far field of RIS), (18) is solved as

$$
L_{\text {bound }} \approx M N \sqrt{\frac{G d_{x} d_{y} F\left(\theta_{t}, \varphi_{t}\right) F\left(\theta_{r}, \varphi_{r}\right)}{4 \pi}} .
$$

For the large RIS1 simulation, $L_{\text {bound }}$ equals to $28.77 \mathrm{~m}$, which is in good agreement with Fig. 10(d). In the rest of the paper, (19) is referred to as the boundary of the far field and the near field for RIS-assisted wireless communications.

2) Intelligent Reflection in the Near Field Broadcasting Case: $P_{t}=0 \mathrm{dBm}, \theta_{t}=\frac{\pi}{4}, \varphi_{t}=\pi$. The large RIS1 and $\mathrm{X}$-band antennas are employed in the simulation. $\phi_{n, m}$ are designed according to (16) for desired broadcasting direction of $\theta_{\text {des }}=\frac{\pi}{4}$ and $\varphi_{\text {des }}=\frac{\pi}{4}$. Fig. 11 (a) shows the received signal power distribution in various directions when $d_{1}=2 \mathrm{~m}$ and $d_{2}=100 \mathrm{~m}$. The large RIS 1 successfully broadcasts the reflected signal into the desired direction. Fig. 11.b) illustrates that the near-field broadcasting formula (14) fits well with the general formula (3), which validates the free-space path loss in (15) for RIS-assisted near-field intelligent broadcasting scenario. The large RIS2 is employed in the simulation when $\theta_{t}=0 . \phi_{n, m}$ are designed to steer the reflected signal towards two desired directions: $\theta_{\text {des }}=\frac{\pi}{4}, \varphi_{\text {des }}=0$ and $\theta_{\text {des }}=\frac{\pi}{4}, \varphi_{\text {des }}=\pi . \phi_{n, m}=0$ when $\bmod (m, 4)=0$ or 1 and $\phi_{n, m}=\pi$ when $\bmod (m, 4)=2$ or 3 [9]. Fig. 11.c) shows the received signal power distribution when $d_{1}=1 \mathrm{~m}$ and $d_{2}$ $=100 \mathrm{~m}$. It can be observed that the large RIS2 successfully shapes the reflected signal towards two desired directions at the same time. As shown in Fig. 11(d), the near-field broadcasting formula (14) fits well with the general formula (3), which reveals the free-space path loss also follows (15) under this scenario.

\section{Path Loss Simulation Summary}

The numerical simulations have verified the free-space path loss models of RIS-assisted wireless communications summarized in Table I via the general formula (3). In addition, the boundary of the far field and the near field of the RIS is redefined in (19), which can be used to determine whether the transmitter/receiver is in the near or far field of the RIS.

\section{VAlidation of Path Loss Models Via EXPERIMENTAL MEASUREMENTS}

Experimental measurements are carried out to further validate the proposed free-space path loss models for RISassisted systems. We set up a path loss measurement system in a microwave anechoic chamber and utilize three different metasurfaces to act as RISs in different scenarios. 


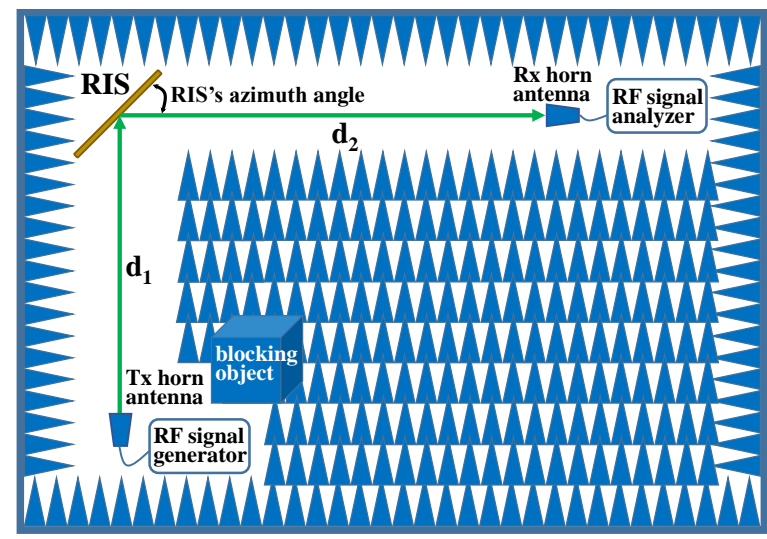

(a)

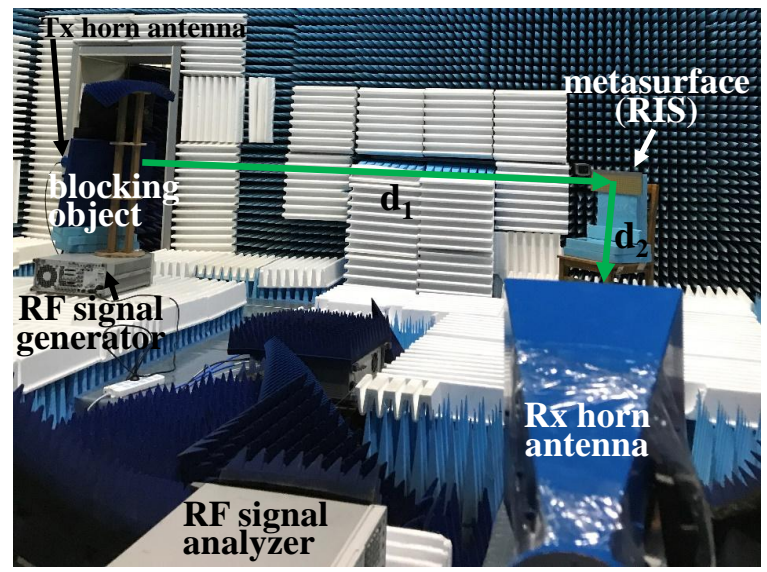

(b)

Fig. 12. Free-space path loss measurement system of RIS-assisted wireless communications: (a) diagram. (b) photo.

\section{A. Measurement Setup}

Fig. 12 illustrates our free-space path loss measurement system for RIS-assisted wireless communications, which is composed of a metasurface, RF signal generator (Agilent E8267D), Tx horn antenna, RF signal analyzer (Agilent N9010A), Rx horn antenna and accessories such as cables and blocking object (electromagnetic wave-absorbing materials). During the experimental measurement, the polarization directions of the metasurface, Tx horn antenna, and Rx horn antenna are well matched, which are all horizontally polarized. The RF signal generator provides the RF signal with a constant power to the Tx horn antenna, which illuminates the metasurface through distance $d_{1}$. The signal reflected by the metasurface propagates over distance $d_{2}$, and is received by the Rx horn antenna and the RF signal analyzer, which gives the measurement result of the received signal power. Because multipath propagation is greatly suppressed in the microwave anechoic chamber and the blocking object cuts off the direct path between the Tx antenna and the Rx antenna, the received signal mainly comes from the reflection of the RIS, which ensures the feasibility of the free-space path loss measurements of RISassisted wireless communications. Since $d_{1}$ and $d_{2}$ need to be flexibly changed during the measurement to study the relationship of the path loss as a function of $d_{1}$ and $d_{2}$, we utilize the two aisles perpendicular to each other in the anechoic chamber and place the metasurface at the junction

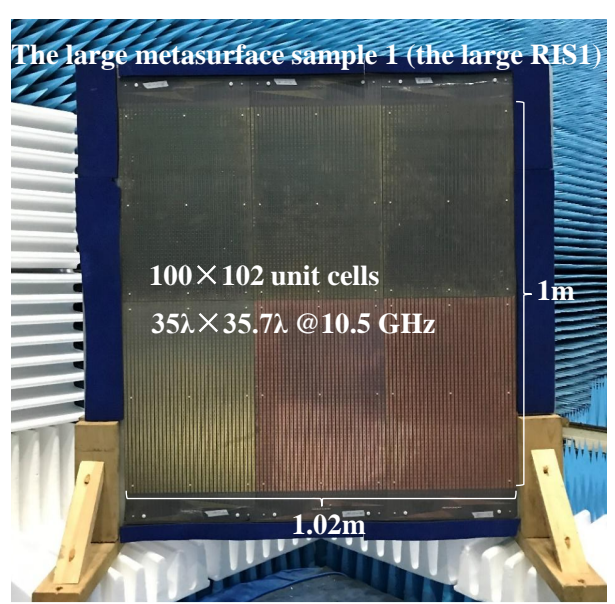

(a)

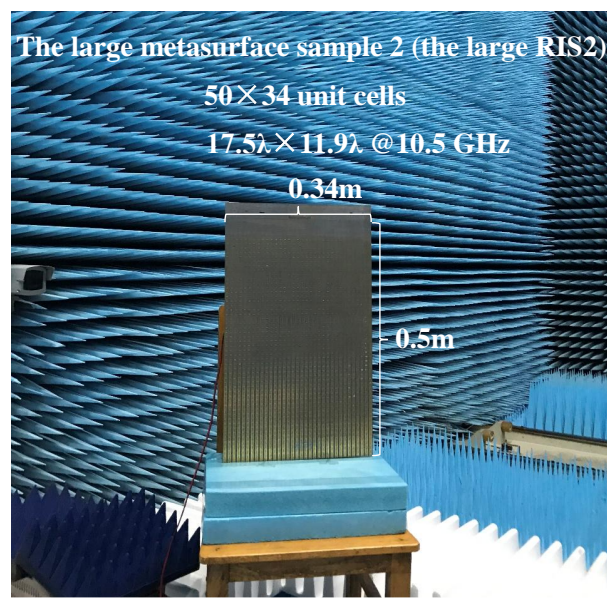

(b)

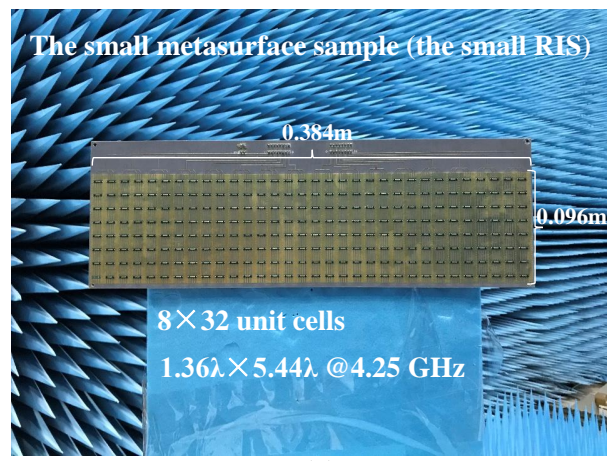

(c)

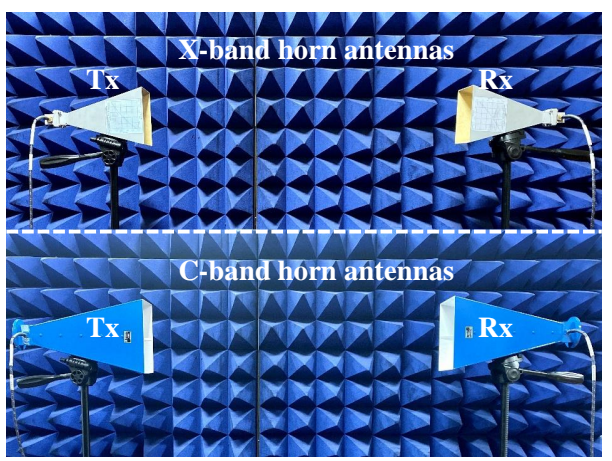

(d)

Fig. 13. Photographs of the metasurfaces and horn antennas utilized for the free-space path loss measurements of RIS-assisted wireless communications. (a) the large RIS1. (b) the large RIS2. (c) the small RIS. (d) horn antennas. 


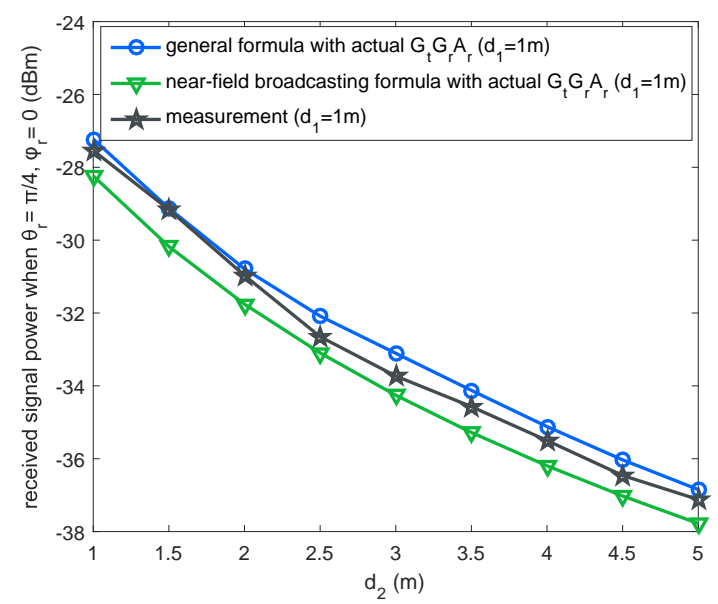

(a)

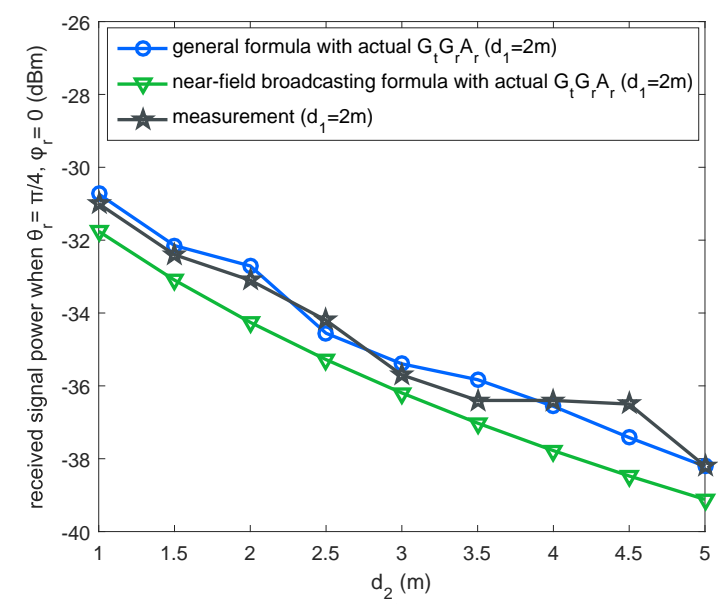

(b)

Fig. 14. Measurement results of specular reflection through the large RIS1 in the near field broadcasting case. (a) received signal power versus $d_{2}$ when $d_{1}$ $=1 \mathrm{~m}$. (b) received signal power versus $d_{2}$ when $d_{1}=2 \mathrm{~m}$.

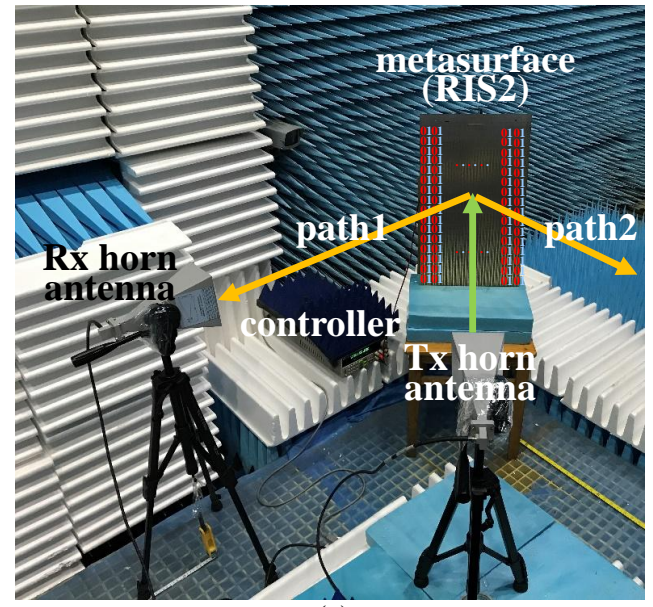

(a)

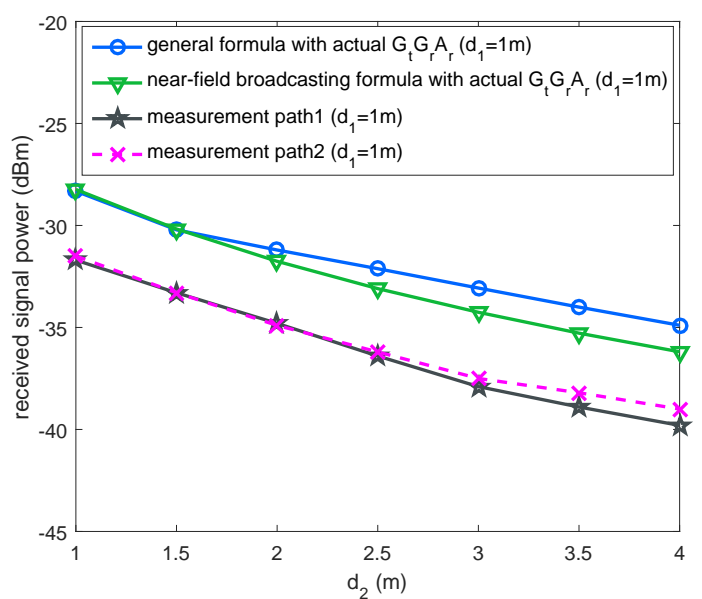

(b)

Fig. 15. Measurement photograph and results of intelligent reflection through the large RIS2 in the near field broadcasting case. (a) Photograph. (b) Received signal power along the two paths versus $d_{2}$ when $d_{1}=1 \mathrm{~m}$.

of the two aisles. The angles between the metasurface and the two aisles are both $45^{\circ}$ (i.e., ideally, RIS's azimuth angle in Fig. 12 a) equals $45^{\circ}$ ), which indicates that the metasurface performs specular reflection for most of our measurements. In addition, we also measured an intelligent reflection scenario, in which the incident wave is perpendicular to the metasurface and the reflected wave is manipulated into two directions. Fig. 13 shows the metasurfaces and horn antennas utilized in the free-space path loss measurements in different scenarios. Their main parameters are consistent with those used for the simulations described in Table $\Pi$ at the beginning of Section IV.

\section{B. Measurement Results}

We utilize the two large RISs and the small RIS to measure the free-space path loss in the RIS-assisted broadcasting and beamforming scenarios, respectively. The $L_{\text {bound }}$ (boundary between the far field and the near field) of the large RIS1, the large RIS2 and the small RIS is $28.77 \mathrm{~m}, 4.8 \mathrm{~m}$ and 0.866 $\mathrm{m}$ according to (19), respectively. This makes it possible to measure the path loss in the near field case of the two large RISs and in the far field case of the small RIS, as the area of the microwave anechoic chamber is about $6 \mathrm{~m} \times 5 \mathrm{~m}$.

\section{1) Specular Reflection of Large RIS1 in the Near Field} Broadcasting Case: Let all the unit cells of the large RIS1 have the same reflection coefficient by applying the same control voltage $(0 \mathrm{~V})$ to them. The transmit power is fixed to $0 \mathrm{dBm}$ and the large RIS1 specularly reflects the incident signal as depicted in Fig 12 a). Fig 14 illustrates the measured received signal power versus $d_{1}$ and $d_{2}$, from which we can observe that the measurement results are in good agreement with the theoretical general formula (3) and the proposed near-field broadcasting formula (14). The measurement results practically validate that the free-space path loss of RIS-assisted wireless communication follows (15) under the scenario of specular reflection through a large RIS in the near field broadcasting case. The path loss is proportional to $\left(d_{1}+d_{2}\right)^{2}$. It should be noted that in order to fairly compare the mea- 


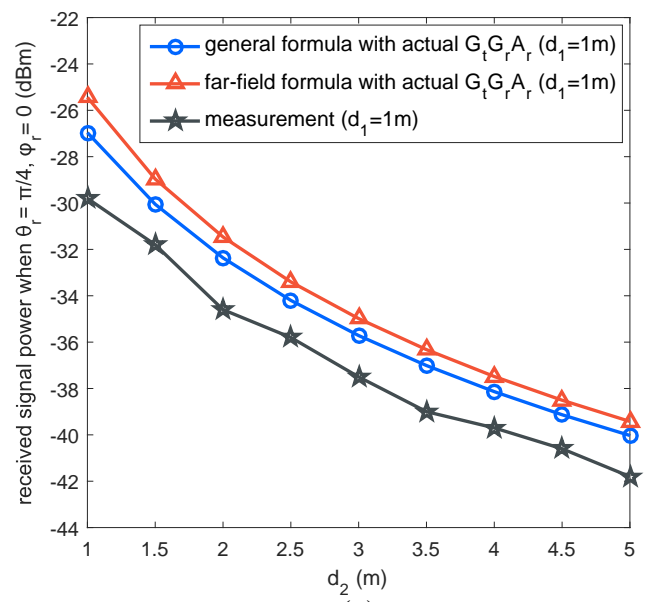

(a)

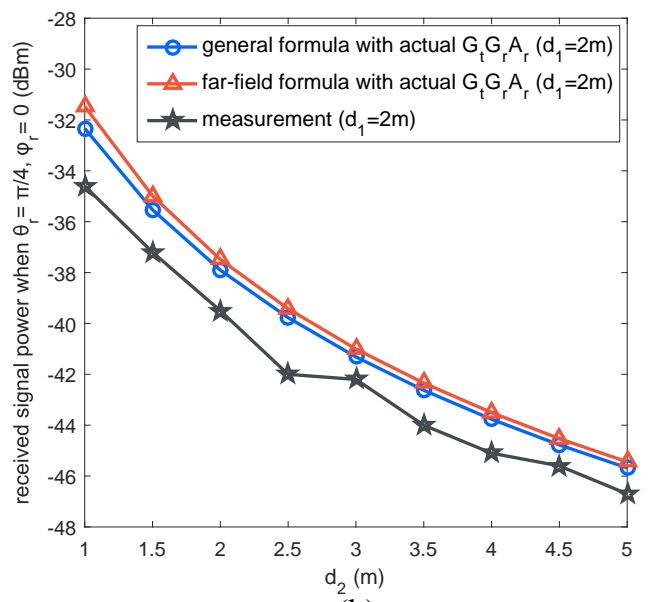

(b)

Fig. 16. Measurement results of specular reflection through the small RIS in the far field beamforming case. (a) Received signal power versus $d_{2}$ when $d_{1}$ $=1 \mathrm{~m}$. (b) Received signal power versus $d_{2}$ when $d_{1}=2 \mathrm{~m}$.

surement results with the theoretical models, we measured the actual value of $G_{t} G_{r} A_{r} G_{\text {lineloss }}\left(G_{\text {lineloss }}\right.$ denotes the cable loss, which is introduced by the RF cables utilized in the measurements) and substituted it into the proposed theoretical formulas.

2) Intelligent Reflection of Large RIS2 in the Near Field Broadcasting Case: The incident wave is perpendicular to the large RIS2 and the reflected wave is reflected towards two paths $\left(\theta_{r}=\frac{\pi}{4}, \varphi_{r}=\pi\right.$ and $\left.\theta_{r}=\frac{\pi}{4}, \varphi_{r}=0\right)$ simultaneously, as sketched in Fig. 15(a). Fig. 15(b) illustrates the measured received signal power along the two paths versus $d_{2}$ when $d_{1}=1 \mathrm{~m}$. We can see that the trend of the two measurement curves are exactly the same as that of the proposed theoretical curves, and the difference between them is only about $3 \mathrm{~dB}{ }^{7}$. The measurement results validate that the free-space path loss of RIS-assisted wireless communication follows (15) under the scenario of intelligent reflection through a large RIS in the near field broadcasting case.

3) Specular Reflection of Small RIS in the Far Field Beamforming Case: Let all the unit cells of the small RIS have the same reflection coefficient by applying the same control voltage $(0 \mathrm{~V})$ to them. The transmit power is fixed to $0 \mathrm{dBm}$ and the small RIS specularly reflects the incident signal to the direction of $\theta_{r}=\frac{\pi}{4}$ and $\varphi_{r}=0$, along which we measured the received signal power as depicted in Fig 12 Fig.16 illustrates the measured received signal power versus $d_{1}$ and $d_{2}$. We observe that the measurement results are in good agreement with the theoretical general formula (3) and the proposed farfield formula (4). The difference between them is only about $2 \mathrm{~dB}$ (see footnote7). The measurement results practically validate that the free-space path loss of RIS-assisted wireless

\footnotetext{
${ }^{7}$ The slight position deviations of the RIS, Tx horn antenna, and Rx horn antenna will lead to the differences between theory and the measurements. Meanwhile, these slight position deviations are hard to avoid in practice. The deviation of the relative position causes the center direction of the reflected beam of the RIS to deviate from the measurement path, which results in lower measurement results. For example, just a very few degrees' deviation on RIS's azimuth angle can cause $2 \sim 3 \mathrm{~dB}$ decrease in the measured received power which can be verified through simulations based on the general formula 3 in Theorem 1 .
}

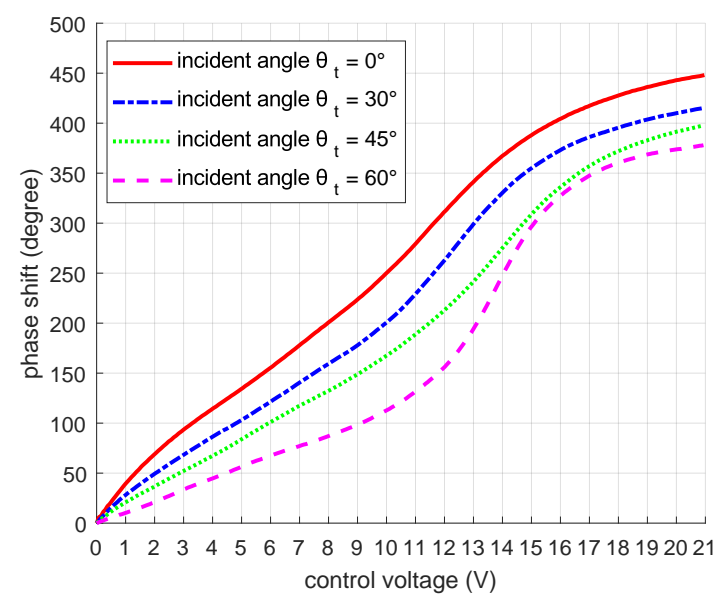

Fig. 17. Relationship between the control voltage and the phase shift of the unit cells of the used small RIS.

communication follows (6) under the scenario of specular reflection through RIS in the far field beamforming case. The free-space path loss is proportional to $\left(d_{1} d_{2}\right)^{2}$ when the RISassisted wireless communications aim to achieve beamforming in the far field case.

4) On the Sensitivity of Phase Regulation to the Incident Angle: The general formula (3) reveals that RIS-assisted wireless communication systems are transmitter-receiver reciprocal, which is based on the assumption that $\Gamma_{n, m}$ is not sensitive to the changes of the incident angle $\theta_{t}$. If $\Gamma_{n, m}$ is sensitive to $\theta_{t}$, especially if its phase $\phi_{n, m}$ is sensitive to $\theta_{t}$, the channel reciprocity of RIS-assisted wireless communications will not hold anymore. We measured the relationship between the control voltage and the phase shift of the unit cells of our small RIS under different incident angles as plotted in Fig. 17. It can be observed that the used RIS is highly sensitive to the incident angle. Therefore, it is necessary to develop metasurfaces that are insensitive to the incident angle, thus ensuring channel reciprocity in RIS-assisted wireless communications. 
5) Power Consumption of the RISs: The utilized small RIS belongs to the family of varactor-diode-based programmable metasurfaces, while the two large RISs belong to the family of PIN-diode-based programmable metasurfaces. The power consumption of varactor-diode-based RISs is almost zero, because the current in the varactor diodes of the unit cells is negligible when it is working. Therefore, the power comsumption of varactor-diode-based RISs is $P_{R I S}^{\text {varactor }} \approx 0$. The power consumption of PIN-diode-based RISs is related to the states of their unit cells. The phase shift of each unit cell can be adjusted between 0 and $\pi$ by electrically controlling the "on" and "off" state of the PIN diode. In the power consumption experiment, $0 \mathrm{~V}$ voltage signal is provided to all unit cells of the large RIS2 by a DC voltage source instrument. In this case, all diodes are in the "off" state, and the current output of the DC voltage source is negligible. On the contrary, when $0.7 \mathrm{~V}$ voltage signal is provided, all diodes are in the "on" state. In this case, the current output of the DC voltage source is $0.8 \mathrm{~A}$, which indicates that the power consumption of each unit cell in the "on" state is $0.33 \mathrm{~mW}(0.7$ $\mathrm{V} * 0.8 \mathrm{~A} /(50 * 34$ unit cells $)=0.33 \mathrm{~mW} /$ unit cell $)$. Therefore, the power comsumption of our designed PIN-diode-based RIS is $P_{R I S}^{P I N}=0.33 N_{\text {on }} m W$, where $N_{\text {on }}$ is the number of unit cells in the "on" state. It should be noted that the above calculations account only for the RISs themselves. In practice, the controller which provides control signals to the RIS also consumes energy, and its power consumption is related to the controller's circuit design and the number of output control signals. The power consumption of the controller we designed for the utilized small RIS is about $0.72 \mathrm{~W}$, and that for the large RIS2 is about $10 \mathrm{~W}$.

\section{CONCLUSION}

In this paper, we have theoretically developed free-space path loss models for RIS-assisted wireless communications based on the electromagnetic and physics properties of the RISs. The general formula that yields the free-space path loss of RIS-assisted wireless communications in different scenarios was first proposed. Subsequently, three more insightful freespace path loss models have been derived, namely the far-field formula, the near-field beamforming formula and the near-field broadcasting formula, to characterize the free-space path loss of RIS-assisted beamforming and broadcasting, respectively. In addition, the boundary of the far field and the near field regimes of RIS-assisted wireless communications has been discussed according to the numerical simulations. Moreover, free-space path loss measurements of RIS-assisted wireless communications have been conducted in a microwave anechoic chamber by considering different scenarios. The measurement results match quite well with the modeling results, which validates the proposed path loss models. This work can help researchers understand the basic large-scale fading of RIS-assisted wireless communication systems, which is useful for link budget calculation and performance analysis. More fading factors, such as small-scale fading, deserve further studies in the future as an important generalization of this paper.

\section{APPENDIX A}

\section{Proof of Theorem 1}

The power of the incident signal into unit cell $U_{n, m}$ can be expressed as

$$
P_{n, m}^{i n}=\frac{G_{t} P_{t}}{4 \pi r_{n, m}^{t}{ }^{2}} F^{t x}\left(\theta_{n, m}^{t x}, \varphi_{n, m}^{t x}\right) F\left(\theta_{n, m}^{t}, \varphi_{n, m}^{t}\right) d_{x} d_{y},
$$

and the electric field of the incident signal into $U_{n, m}$ is given by

$$
E_{n, m}^{i n}=\sqrt{\frac{2 Z_{0} P_{n, m}^{i n}}{d_{x} d_{y}}} e^{\frac{-j 2 \pi r_{n, m}^{t}}{\lambda}},
$$

where $Z_{0}$ is the characteristic impedance of the air, and $r_{n, m}^{t}$ can be written as

$$
r_{n, m}^{t}=\sqrt{\left(x_{t}-\left(m-\frac{1}{2}\right) d_{x}\right)^{2}+\left(y_{t}-\left(n-\frac{1}{2}\right) d_{y}\right)^{2}+z_{t}{ }^{2}} .
$$

According to the law of energy conservation, for the unit cell $U_{n, m}$, the power of the incident signal times the square of the reflection coefficient is equal to the total power of the reflected signal, thus we have

$$
P_{n, m}^{i n}\left|\Gamma_{n, m}^{2}\right|=P_{n, m}^{r e f l e c t},
$$

where $P_{n, m}^{\text {reflect }}$ is the total reflected signal power of the unit cell $U_{n, m}$, and the reflection coefficient can be written as

$$
\Gamma_{n, m}=A_{n, m} e^{j \phi_{n, m}},
$$

where $A_{n, m}$ and $\phi_{n, m}$ represent the controllable amplitude and phase shift of $U_{n, m}$, respectively.

The power of the reflected signal received by the receiver from $U_{n, m}$ can be expressed as

$$
P_{n, m}^{r}=\frac{G P_{n, m}^{r e f l e c t}}{4 \pi r_{n, m}^{r}{ }^{2}} F\left(\theta_{n, m}^{r}, \varphi_{n, m}^{r}\right) F^{r x}\left(\theta_{n, m}^{r x}, \varphi_{n, m}^{r x}\right) A_{r},
$$

where $A_{r}$ represents the aperture of the receiving antenna, and $r_{n, m}^{r}$ can be written as

$$
r_{n, m}^{r}=\sqrt{\left(x_{r}-\left(m-\frac{1}{2}\right) d_{x}\right)^{2}+\left(y_{r}-\left(n-\frac{1}{2}\right) d_{y}\right)^{2}+z_{r}{ }^{2}} .
$$

By combining (20), (23) and (25), the electric field of the reflected signal received by the receiver from $U_{n, m}$ is obtained as

$$
\begin{aligned}
& E_{n, m}^{r}=\sqrt{\frac{2 Z_{0} P_{n, m}^{r}}{A_{r}}} e^{-j\left(\frac{2 \pi r_{n, m}^{t}}{\lambda}+\frac{2 \pi r_{n, m}^{r}}{\lambda}-\phi_{n, m}\right)} \\
& =\sqrt{\frac{Z_{0} P_{t} G_{t} G d_{x} d_{y} F_{n, m}^{c o m b i n e}}{8 \pi^{2}}} \frac{\Gamma_{n, m}}{r_{n, m}^{t} r_{n, m}^{r}} e^{\frac{-j 2 \pi\left(r_{n, m}^{t}+r_{n, m}^{r}\right)}{\lambda}},
\end{aligned}
$$

where $-\left(\frac{2 \pi r_{n, m}^{t}}{\lambda}+\frac{2 \pi r_{n, m}^{r}}{\lambda}-\phi_{n, m}\right)$ is the phase alteration caused by the propagation and the reflection coefficient of $U_{n, m}$. The total electric field of the received signal is the 
superposition of the electric fields reflected by all unit cells towards the receiver, which can be written as [45]-[47]

$$
E^{r}=\sum_{m=1-\frac{M}{2}}^{\frac{M}{2}} \sum_{n=1-\frac{N}{2}}^{\frac{N}{2}} E_{n, m}^{r} .
$$

The received signal power of the receiver is

$$
P_{r}=\frac{\left|E^{r}\right|^{2}}{2 Z_{0}} A_{r},
$$

where the aperture of the receiving antenna can be written as

$$
A_{r}=\frac{G_{r} \lambda^{2}}{4 \pi} .
$$

We obtain Theorem 1 by substituting (27), 28), and 30 ) into (29).

\section{APPENDIX B}

Proof of Proposition 1

The position of the transmitter in Fig. 2 is

$$
\left(x_{t}, y_{t}, z_{t}\right)=\left(d_{1} \sin \theta_{t} \cos \varphi_{t}, d_{1} \sin \theta_{t} \sin \varphi_{t}, d_{1} \cos \theta_{t}\right) .
$$

By combining (22) and (31), when the transmitter is in the far field of the RIS, $r_{n, m}^{t}$ can be further expressed as 32 shown at the top of the next page.

Similarly, the position of the receiver in Fig. 2 is

$$
\left(x_{r}, y_{r}, z_{r}\right)=\left(d_{2} \sin \theta_{r} \cos \varphi_{r}, d_{2} \sin \theta_{r} \sin \varphi_{r}, d_{2} \cos \theta_{r}\right) .
$$

By combining (26) and (33), when the receiver is in the far field of the RIS, $r_{n, m}^{r}$ can be further expressed as 34p shown at the top of the next page.

In the far field case, $\left(\theta_{n, m}^{t}, \varphi_{n, m}^{t}\right)$ and $\left(\theta_{n, m}^{r}, \varphi_{n, m}^{r}\right)$ can be approximated as $\left(\theta_{t}, \varphi_{t}\right)$ and $\left(\theta_{r}, \varphi_{r}\right)$, respectively. In addition, as assuming that the direction of peak radiation of both the transmitting antenna and receiving antenna point to the center of the RIS, we have $F^{t x}\left(\theta_{n, m}^{t x}, \varphi_{n, m}^{t x}\right) \approx 1$ and $F^{r x}\left(\theta_{n, m}^{r x}, \varphi_{n, m}^{r x}\right) \approx 1$ in the far field case.

By substituting (32) and 34) into the general formula (3), the received signal power can be further written as

$$
\begin{aligned}
P_{r} & \approx P_{t} \frac{G_{t} G_{r} G d_{x} d_{y} \lambda^{2}}{64 \pi^{3}} \\
& \times\left|\sum_{m=1-\frac{M}{2}}^{\frac{M}{2}} \sum_{n=1-\frac{N}{2}}^{\frac{N}{2}} \sqrt{F\left(\theta_{t}, \varphi_{t}\right) F\left(\theta_{r}, \varphi_{r}\right)} \Gamma_{n, m} \frac{e^{\frac{-j 2 \pi \alpha}{\lambda}}}{d_{1} d_{2}}\right|^{2} \\
= & P_{t} \frac{G_{t} G_{r} G d_{x} d_{y} \lambda^{2} F\left(\theta_{t}, \varphi_{t}\right) F\left(\theta_{r}, \varphi_{r}\right)}{64 \pi^{3} d_{1}^{2} d_{2}^{2}} \\
& \times\left|\sum_{m=1-\frac{M}{2}}^{\frac{M}{2}} \sum_{n=1-\frac{N}{2}}^{\frac{N}{2}} \Gamma_{n, m} e^{\frac{j 2 \pi\left(d_{1}+d_{2}-\alpha\right)}{\lambda}}\right|^{2},
\end{aligned}
$$

where $\alpha=d_{1}-\sin \theta_{t} \cos \varphi_{t}\left(m-\frac{1}{2}\right) d_{x}-\sin \theta_{t} \sin \varphi_{t}\left(n-\frac{1}{2}\right) d_{y}+$ $d_{2}-\sin \theta_{r} \cos \varphi_{r}\left(m-\frac{1}{2}\right) d_{x}-\sin \theta_{r} \sin \varphi_{r}\left(n-\frac{1}{2}\right) d_{y}$.

As all the unit cells of RIS share the same reflection coefficient $A e^{j \phi}, 35$ can be written as

$$
P_{r}=P_{t} \frac{G_{t} G_{r} G d_{x} d_{y} \lambda^{2} F\left(\theta_{t}, \varphi_{t}\right) F\left(\theta_{r}, \varphi_{r}\right) A^{2}}{64 \pi^{3} d_{1}{ }^{2} d_{2}{ }^{2}}|\beta|^{2},
$$

where $\beta$ is defined as 37 shown at the top of the next page.

Define $u=\frac{2 \pi}{\lambda}\left(\sin \theta_{t} \cos \varphi_{t}+\sin \theta_{r} \cos \varphi_{r}\right) d_{x}$ and $v=$ $\frac{2 \pi}{\lambda}\left(\sin \theta_{t} \sin \varphi_{t}+\sin \theta_{r} \sin \varphi_{r}\right) d_{y}$, then 37p can be rewritten as

$$
\begin{aligned}
\beta & =\sum_{m=1-\frac{M}{2}}^{\frac{M}{2}} \sum_{n=1-\frac{N}{2}}^{\frac{N}{2}} e^{j\left(m-\frac{1}{2}\right) u} e^{j\left(n-\frac{1}{2}\right) v} \\
& =\sum_{m=1-\frac{M}{2}}^{\frac{M}{2}} e^{j\left(m-\frac{1}{2}\right) u} \sum_{n=1-\frac{N}{2}}^{\frac{N}{2}} e^{j\left(n-\frac{1}{2}\right) v}
\end{aligned}
$$

By applying the formula of the sum of the geometric progression, 38 can be rewritten as

$$
\begin{aligned}
\beta= & \frac{e^{-j \frac{M u}{2}}-e^{j \frac{M u}{2}}}{e^{-j \frac{u}{2}}-e^{j \frac{u}{2}}} \frac{e^{-j \frac{N v}{2}}-e^{j \frac{N v}{2}}}{e^{-j \frac{v}{2}}-e^{j \frac{v}{2}}} \\
= & \frac{\sin \left(\frac{M u}{2}\right)}{\sin \left(\frac{u}{2}\right)} \frac{\sin \left(\frac{N v}{2}\right)}{\sin \left(\frac{v}{2}\right)}=M N \frac{\operatorname{sinc}\left(\frac{M u}{2}\right)}{\operatorname{sinc}\left(\frac{u}{2}\right)} \frac{\operatorname{sinc}\left(\frac{N v}{2}\right)}{\operatorname{sinc}\left(\frac{v}{2}\right)} \\
= & M N \frac{\operatorname{sinc}\left(\frac{M \pi}{\lambda}\left(\sin \theta_{t} \cos \varphi_{t}+\sin \theta_{r} \cos \varphi_{r}\right) d_{x}\right)}{\operatorname{sinc}\left(\frac{\pi}{\lambda}\left(\sin \theta_{t} \cos \varphi_{t}+\sin \theta_{r} \cos \varphi_{r}\right) d_{x}\right)} \\
& \times \frac{\operatorname{sinc}\left(\frac{N \pi}{\lambda}\left(\sin \theta_{t} \sin \varphi_{t}+\sin \theta_{r} \sin \varphi_{r}\right) d_{y}\right)}{\operatorname{sinc}\left(\frac{\pi}{\lambda}\left(\sin \theta_{t} \sin \varphi_{t}+\sin \theta_{r} \sin \varphi_{r}\right) d_{y}\right)} .
\end{aligned}
$$

By plugging (39) into 36, the received signal power in the far field case can be obtained as stated in (4). When $\sin \theta_{t} \cos \varphi_{t}+\sin \theta_{r} \cos \varphi_{r}=0$ and $\sin \theta_{t} \sin \varphi_{t}+$ $\sin \theta_{r} \sin \varphi_{r}=0$, that is, $\theta_{r}=\theta_{t}$ and $\varphi_{r}=\varphi_{t}+\pi$, (4) is maximized as stated in (5).

\section{APPENDIX C}

Proof of Proposition 2

For the intelligent reflection in the far field case, by substituting $\Gamma_{n, m}=A e^{j \phi_{n, m}}$ into 35 , we have

$$
P_{r}=P_{t} \frac{G_{t} G_{r} G d_{x} d_{y} \lambda^{2} F\left(\theta_{t}, \varphi_{t}\right) F\left(\theta_{r}, \varphi_{r}\right) A^{2}}{64 \pi^{3} d_{1}^{2} d_{2}^{2}}|\tilde{\beta}|^{2},
$$

where $\tilde{\beta}$ is defined as 41 shown at the top of the next page.

Define $\tilde{u}=\frac{2 \pi}{\lambda}\left(\sin \theta_{t} \cos \varphi_{t}+\sin \theta_{r} \cos \varphi_{r}+\delta_{1}\right) d_{x}$ and $\tilde{v}=$ $\frac{2 \pi}{\lambda}\left(\sin \theta_{t} \sin \varphi_{t}+\sin \theta_{r} \sin \varphi_{r}+\delta_{2}\right) d_{y}$, where $\delta_{1}\left(m-\frac{1}{2}\right) d_{x}+$ $\delta_{2}\left(n-\frac{1}{2}\right) d_{y}=\frac{\lambda \phi_{n, m}}{2 \pi}, 41$ can be rewritten as

$$
\begin{aligned}
\tilde{\beta} & =\sum_{m=1-\frac{M}{2}}^{\frac{M}{2}} \sum_{n=1-\frac{N}{2}}^{\frac{N}{2}} e^{j\left(m-\frac{1}{2}\right) \tilde{u}} e^{j\left(n-\frac{1}{2}\right) \tilde{v}} \\
& =\sum_{m=1-\frac{M}{2}}^{\frac{M}{2}} e^{j\left(m-\frac{1}{2}\right) \tilde{u}} \sum_{n=1-\frac{N}{2}}^{\frac{N}{2}} e^{j\left(n-\frac{1}{2}\right) \tilde{v}}
\end{aligned}
$$

Similarly, by applying the formula of the sum of the geometric progression, the received signal power can be obtained as (7), which is maximized when $\sin \theta_{t} \cos \varphi_{t}+\sin \theta_{r} \cos \varphi_{r}+$ $\delta_{1}=0$ and $\sin \theta_{t} \sin \varphi_{t}+\sin \theta_{r} \sin \varphi_{r}+\delta_{2}=0$.

\section{APPENDIX D}

\section{Proof of Proposition 3}

By substituting $\Gamma_{n, m}=A e^{j \phi_{n, m}}$ into the general formula (3), (10) can be simply obtained. For the desired receiver at $\left(x_{r}, y_{r}, z_{r}\right), 10$ is maximized as 11$]$ when $2 \pi\left(r_{n, m}^{t}+r_{n, m}^{r}\right)-$ $\lambda \phi_{n, m}=0$, which can be further expressed as 12$]$. 


$$
\begin{aligned}
r_{n, m}^{t} & =\sqrt{\left(d_{1} \sin \theta_{t} \cos \varphi_{t}-\left(m-\frac{1}{2}\right) d_{x}\right)^{2}+\left(d_{1} \sin \theta_{t} \sin \varphi_{t}-\left(n-\frac{1}{2}\right) d_{y}\right)^{2}+\left(d_{1} \cos \theta_{t}\right)^{2}} \\
& \approx d_{1}-\sin \theta_{t} \cos \varphi_{t}\left(m-\frac{1}{2}\right) d_{x}-\sin \theta_{t} \sin \varphi_{t}\left(n-\frac{1}{2}\right) d_{y},
\end{aligned}
$$

$$
\begin{aligned}
r_{n, m}^{r} & =\sqrt{\left(d_{2} \sin \theta_{r} \cos \varphi_{r}-\left(m-\frac{1}{2}\right) d_{x}\right)^{2}+\left(d_{2} \sin \theta_{r} \sin \varphi_{r}-\left(n-\frac{1}{2}\right) d_{y}\right)^{2}+\left(d_{2} \cos \theta_{r}\right)^{2}} \\
& \approx d_{2}-\sin \theta_{r} \cos \varphi_{r}\left(m-\frac{1}{2}\right) d_{x}-\sin \theta_{r} \sin \varphi_{r}\left(n-\frac{1}{2}\right) d_{y},
\end{aligned}
$$

$$
\beta=\sum_{m=1-\frac{M}{2}}^{\frac{M}{2}} \sum_{n=1-\frac{N}{2}}^{\frac{N}{2}} e^{\frac{j 2 \pi\left(\left(\sin \theta_{t} \cos \varphi_{t}+\sin \theta_{r} \cos \varphi_{r}\right)\left(m-\frac{1}{2}\right) d_{x}+\left(\sin \theta_{t} \sin \varphi_{t}+\sin \theta_{r} \sin \varphi_{r}\right)\left(n-\frac{1}{2}\right) d_{y}\right)}{\lambda}}
$$

$$
\tilde{\beta}=\sum_{m=1-\frac{M}{2}}^{\frac{M}{2}} \sum_{n=1-\frac{N}{2}}^{\frac{N}{2}} e^{\frac{j 2 \pi\left(\left(\sin \theta_{t} \cos \varphi_{t}+\sin \theta_{r} \cos \varphi_{r}\right)\left(m-\frac{1}{2}\right) d_{x}+\left(\sin \theta_{t} \sin \varphi_{t}+\sin \theta_{r} \sin \varphi_{r}\right)\left(n-\frac{1}{2}\right) d_{y}+\frac{\lambda \phi_{n, m}}{2 \pi}\right)}{\lambda}} .
$$

\section{REFERENCES}

[1] Huawei, "Asia-Pacific leads 5G innovation, Huawei enables sustainable development of a digital economy," Sep. 2019. [Online]. Available: https://www.huawei.com/en/press-events/news/2019/9/huawei-5thasia-pacific-innovation-day.

[2] T. S. Rappaport et al., "Wireless communications and applications above $100 \mathrm{GHz}$ : Opportunities and challenges for $6 \mathrm{G}$ and beyond," IEEE Access, vol. 7, pp. 78729-78757, Jun. 2019.

[3] Y. Zeng and R. Zhang, "Millimeter wave MIMO with lens antenna array: A new path division multiplexing paradigm," IEEE Trans. Commun., vol. 64, no. 4, pp. 1557-1571, Apr. 2016.

[4] F. Tariq, M. R. A. Khandaker, K.-K. Wong, M. Imran, M. Bennis, and M. Debbah, "A speculative study on 6G," IEEE Wireless Commun., vol. 27, no. 4, pp. 118-125, Aug. 2020.

[5] I. F. Akyildiz, C. Han, and S. Nie, "Combating the distance problem in the millimeter wave and terahertz frequency bands," IEEE Commun. Mag., vol. 56, no. 6, pp. 102-108, Jun. 2018.

[6] C. Han, J. M. Jornet, and I. F. Akyildiz, "Ultra-massive MIMO channel modeling for graphene-Enabled terahertz-band communications," in Proc. IEEE VTC Spring, Jun. 2018, pp. 1-5.

[7] S. Hu, F. Rusek, and O. Edfors, "Beyond massive MIMO: The potential of data transmission with large intelligent surfaces," IEEE Trans. Signal Process., vol. 66, no. 10, pp. 2746-2758, May. 2018.

[8] Z. Zhang et al., "6G wireless networks: Vision, requirements, architecture, and key technologies," Vehicular Technology Magazine, vol. 14, no. 3, pp. 28-41, Sep. 2019.

[9] T. J. Cui et al., "Information metamaterials and metasurfaces," J. Mater Chem. C., no. 15, pp. 3644-3668, May 2017.

[10] J. Zhao et al., "Programmable time-domain digital-coding metasurface for non-linear harmonic manipulation and new wireless communication systems," National Sci. Rev., vol. 6, no. 2, pp. 231-238, Mar. 2019.

[11] L. Zhang et al., "Space-time-coding digital metasurfaces," Nat. Commun., vol. 9, Oct. 2018

[12] W. Tang, X. Li, J. Y. Dai, S. Jin, Y. Zeng, Q. Cheng, and T. J. Cui, "Wireless communications with programmable metasurface: Transceiver design and experimental results," China Commun., vol. 16, no. 5, pp. 46-61, May 2019.

[13] W. Tang, J. Y. Dai, M. Chen, X. Li, Q. Cheng, S. Jin, K.-K. Wong, and T. J. Cui, "Programmable metasurface-based RF chain-free 8PSK wireless transmitter," Electron. Lett., vol. 55, no. 7, pp. 417-420, Apr. 2019.
[14] W. Tang et al., "Wireless communications with programmable metasurface: New paradigms, opportunities, and challenges on transceiver design," IEEE Wireless Commun., vol. 27, no. 2, pp. 180-187, Apr. 2020.

[15] J. Y. Dai et al., "Wireless communications through a simplified architecture based on time-domain digital coding metasurface," Adv. Mater. Technol., vol. 4, no. 7, pp. 1-8, Jul. 2019.

[16] M. Di Renzo et al., "Smart radio environments empowered by reconfigurable AI meta-surfaces: An idea whose time has come," EURASIP J. Wireless Commun. Netw., vol. 2019, no. 1, pp. 1-20, May 2019.

[17] Q. Wu and R. Zhang, "Towards smart and reconfigurable environment: Intelligent reflecting surface aided wireless network," IEEE Commun. Mag., vol. 58, no. 1, pp. 106-112, Jan. 2020.

[18] C. Liaskos, S. Nie, A. Tsioliaridou, A. Pitsillides, S. Ioannidis, and I. F Akyildiz, "A new wireless communication paradigm through softwarecontrolled metasurfaces," IEEE Commun. Mag., vol. 56, no. 9, pp. 162169, Sep. 2018.

[19] E. Basar, M. Di Renzo, J. De Rosny, M. Debbah, M. Alouini, and R. Zhang, "Wireless communications through reconfigurable intelligent surfaces," IEEE Access, vol. 7, pp. 116753-116773, Aug. 2019.

[20] Q. Wu and R. Zhang, "Intelligent reflecting surface enhanced wireless network via joint active and passive beamforming," IEEE Trans. Wireless Commun., vol. 18, no. 11, pp. 5394-5409, Nov. 2019.

[21] M. Cui, G. Zhang and R. Zhang, "Secure wireless communication via intelligent reflecting surface," IEEE Wireless Commun. Lett., vol. 8, no. 5, pp. 1410-1414, Oct. 2019.

[22] J. Chen, Y.-C. Liang, Y. Pei and H. Guo, "Intelligent reflecting surface: A programmable wireless environment for physical layer security," IEEE Access, vol. 7, pp. 82599-82612, Jun. 2019.

[23] M. Jung, W. Saas, M. Debbah, and C. S. Hong "On the optimality of reconfigurable intelligent surfaces (RISs): Passive beamforming, modulation, and resource allocation," [Online]. Available: https://arxiv.org/abs/1910.00968.

[24] S. Li, B. Duo, X. Yuan, Y.-C. Liang, and M. D. Renzo, "Reconfigurable intelligent surface assisted UAV communication: Joint trajectory design and passive beamforming" IEEE Wireless Commun. Lett., vol. 9, no. 5, pp. 716-720, May 2020.

[25] H. Guo, Y.-C. Liang, J. Chen, and E. G. Larsson, "Weighted sumrate maximization for intelligent reflecting surface enhanced wireless networks," in Proc. IEEE Global Commun. Conf., Waikoloa, USA, Dec. 2019, pp. 1-6.

[26] C. Huang, A. Zappone, G. C. Alexandropoulos, M. Debbah, and C. Yuen, "Reconfigurable intelligent surfaces for energy efficiency in wireless communication," IEEE Trans. Wireless Commun., vol. 18, no. 8, pp. 4157-4170, Aug. 2019 
[27] C. Huang, G. C. Alexandropoulos, A. Zappone, M. Debbah, and C. Yuen, "Energy efficient multi-user MISO communication using low resolution large intelligent surfaces," in Proc. IEEE Global Commun. Conf., Abu Dhabi, UAE, Dec. 2018.

[28] C. Pan et al., "Intelligent reflecting surface aided MIMO broadcasting for simultaneous wireless information and power transfer," IEEE J. Sel. Areas Commun., vol. 38, no. 8, pp. 1719-1734, Aug. 2020.

[29] Y. Han, W. Tang, S. Jin, C.-K. Wen, and X. Ma, "Large intelligent surface-assisted wireless communication exploiting statistical CSI," IEEE Trans. Veh. Technol., vol. 68, no. 8, pp. 8238-8242, Aug. 2019.

[30] E. Basar, "Transmission through large intelligent surfaces: A new frontier in wireless communications," in Proc. Eur. Conf. Netw. Commun. (EuCNC), Valencia, Spain, Jun. 2019, pp. 112-117.

[31] M. Jung, W. Saad, Y. Jang, G. Kong and S. Choi, "Reliability analysis of large intelligent surfaces (LISs): Rate distribution and outage probability," IEEE Wireless Commun. Lett., vol. 8, no. 6, pp. 1662-1666, Dec. 2019.

[32] M. Di Renzo et al., "Reconfigurable intelligent surfaces vs. relaying: Differences, similarities, and performance comparison," IEEE Open Journal of the Commun. Society, vol. 1, pp. 798-807, Jun. 2020.

[33] Q. Wu and R. Zhang, "Beamforming optimization for intelligent reflecting surface with discrete phase shifts," in Proc. IEEE Int. Conf. Acoust., Speech Signal Process. (ICASSP), Brighton, U.K., May 2019, pp. 78307833.

[34] S. Abeywickrama, R. Zhang, Q. Wu, and C. Yuen, "Intelligent reflecting surface: Practical phase shift model and beamforming optimization," IEEE Trans. Commun., early access, Jun. 2020.

[35] Z.-Q. He and X. Yuan, "Cascaded channel estimation for large intelligent metasurface assisted Massive MIMO," IEEE Wireless Commun. Lett. vol. 9, no. 2, pp. 210-214, Feb. 2020.

[36] A. Taha, M. Alrabeiah, and A. Alkhateeb, "Enabling large intelligent surfaces with compressive sensing and deep learning," [Online]. Available: https://arxiv.org/abs/1904.10136.

[37] B. Zheng and R. Zhang, "Intelligent reflecting surface-enhanced OFDM: Channel estimation and reflection optimization," IEEE Wireless Commun. Lett., vol. 9, no. 4, pp. 518-522, Apr. 2020.

[38] A. Zappone, M. Di Renzo, F. Shams, X. Qian, and M. Debbah, "Overhead-aware design of reconfigurable intelligent surfaces in smart radio environments," [Online]. Available: https://arxiv.org/abs/2003.02538.

[39] Y. Huang and K. Boyle, Antennas: from theory to practice. Chichester: Wiley, 2008

[40] W. L. Stutzman and G. A. Thiele, Antenna theory and design, 3rd Ed., New York: Wiley, 2012.

[41] A. S. Y. Poon, R. W. Brodersen, and D. N. C. Tse, "Degrees of freedom in multiple-antenna channels: A signal space approach," IEEE Trans. Inform. Theory, vol. 51, no. 2, pp. 523-536, Feb. 2005.

[42] A. Goldsmith, Wireless Communications. Cambridge, U.K. : Cambridge University Press, 2005.

[43] J. Zhao et al., "Controlling the bandwidth of terahertz low-scattering metasurfaces," Adv. Optical Mater, vol. 4, no. 11, pp. 1773-1779, Nov. 2016.

[44] L. H. Gao et al., "Broadband diffusion of terahertz waves by multi-bit coding metasurfaces," Light-Sci. Appl., vol. 4, pp. 1-9, Sep. 2015.

[45] H. Chou, T. Hung, N. Wang, H. Chou, C. Tung, and P. Nepa, "Design of a near-field focused reflectarray antenna for $2.4 \mathrm{GHz}$ RFID reader applications," IEEE Trans. Antennas Propag., vol. 59, no. 3, pp. 10131018, Mar. 2011.

[46] S. Yu, H. Liu, and L. Li, "Design of near-field focused metasurface for high-efficient wireless power transfer with multifocus characteristics," IEEE Trans. Ind. Electron., vol. 66, no. 5, pp. 3993-4002, May. 2019.

[47] M. D. Migliore, "A compressed sensing approach for array diagnosis from a small set of near-field measurements," IEEE Trans. Antennas Propag., vol. 59, no. 6, pp. 2127-2133, Jun. 2011

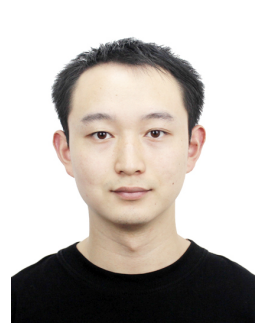

Wankai Tang (S'20) received the B.S. degree in information engineering and the M.S. degree in circuits and systems from Southeast University, Nanjing, China, in 2011 and 2014, respectively. After that, he worked at National Instruments, Shanghai, first as an Applications Engineer and then an Inside Sales Engineer. He is currently pursuing the Ph.D. degree with the National Mobile Communications Research Laboratory, Southeast University. His research interests include modeling and prototyping of metasurface-based wireless communication systems.

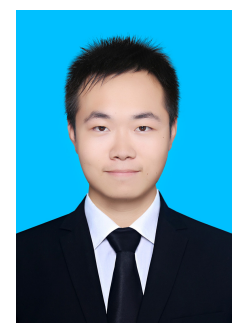

Ming Zheng Chen received the B.S. degree in information engineering from the School of Information Science and Engineering, Southeast University, Nanjing, China, in 2018. He is currently pursuing the M.S. degree with the State Key Laboratory of Millimeter Waves, Southeast University. His research interests include metamaterials, metasurfaces, signal processing and communication systems.

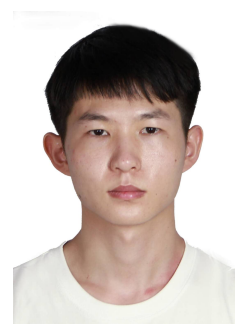

Xiangyu Chen received the B.S. degree in information engineering from the School of Information Science and Engineering, Southeast University, Nanjing, China, in 2019. He is currently pursuing the M.S. degree with the National Mobile Communications Research Laboratory. His research interests include metasurface-based wireless communication system design and measurement.

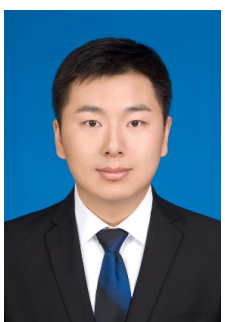

Jun Yan Dai received the B.S. and the Ph.D degrees from Southeast University, Nanjing, China, in 2013 and 2019, respectively. He is currently a Post-Doctoral Fellow with the State Key Laboratory of Terahertz and Millimeter Wave, City University of Hong Kong, Hong Kong. His research interests include metamaterials, metasurfaces, array antennas, and space-time modulation technology.

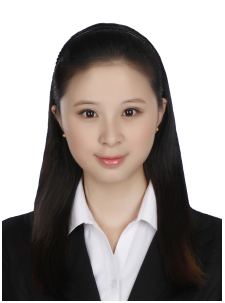

Yu Han (S'14) received the B.S. degree in communications engineering from Hangzhou Dianzi University, Hangzhou, China, in 2012, and the M.S. degree in communications and information systems from Southeast University, Nanjing, China, in 2015, and the Ph.D degree in communications and information systems from Southeast University, Nanjing, China, in 2020. Her research interests include massive MIMO and reconfigurable intelligent surface. 


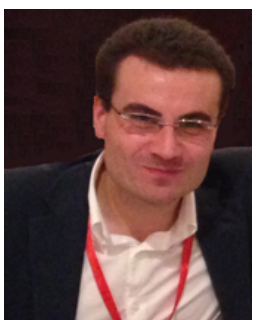

Marco Di Renzo (S'05-AM'07-M'09-SM'14-F'20) was born in L'Aquila, Italy, in 1978. He received the Laurea (cum laude) and Ph.D. degrees in electrical engineering from University of L'Aquila, Italy, in 2003 and 2007, respectively, and the Habilitation a Diriger des Recherches (Doctor of Science) degree from University Paris-Sud, France, in 2013.

Since 2010, he has been with the French National Center for Scientific Research (CNRS), where he is a CNRS Research Director (CNRS Professor) in the Laboratory of Signals and Systems (L2S) of Paris-Saclay University - CNRS and CentraleSupelec, Paris, France. He is a Nokia Foundation Visiting Professor at Aalto University, Finland, and was a Honorary Professor at University Technology Sydney, Sydney, Australia, and a Visiting Professor at the University of L'Aquila, Italy.

$\mathrm{He}$ serves as the Editor-in-Chief of IEEE Communications Letters. He served as an Editor of IEEE Transactions on Communications, IEEE Transactions on Wireless Communications, IEEE Communications Letters, and as the Associate Editor-in-Chief of IEEE Communications Letters. He is a Distinguished Lecturer of the IEEE Vehicular Technology Society and IEEE Communications Society.

$\mathrm{He}$ is a recipient of several awards, including the 2013 IEEE-COMSOC Best Young Researcher Award for Europe, Middle East and Africa, the 2013 NoE-NEWCOM\# Best Paper Award, the 2014-2015 Royal Academy of Engineering Distinguished Visiting Fellowship, the 2015 IEEE Jack Neubauer Memorial Best System Paper Award, the 2015 CNRS Award for Excellence in Research and Ph.D. Supervision, the 2016 MSCA Global Fellowship (declined), the 2017 SEE-IEEE Alain Glavieux Award, the 2018 IEEE-COMSOC Young Professional in Academia Award, the 2019 Nokia Foundation Visiting Professorship, and 8 Best Paper Awards at IEEE conferences (2012 and 2014 IEEE CAMAD, 2013 IEEE VTC-Fall, 2014 IEEE ATC, 2015 IEEE ComManTel, 2017 IEEE SigTelCom, EAI 2018 INISCOM, IEEE ICC 2019).

$\mathrm{He}$ is a Highly Cited Researcher (Clarivate Analytics, Web of Science) and a Fellow of the IEEE.

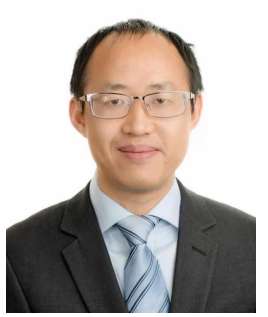

Yong Zeng (S'12-M'14) is with the National Mobile Communications Research Laboratory, Southeast University, Nanjing, China. He received the Bachelor of Engineering (First-Class Honours) and Ph.D. degrees from Nanyang Technological University, Singapore, in 2009 and 2014, respectively. From 2013 to 2018, he was a Research Fellow and Senior Research Fellow at the Department of Electrical and Computer Engineering, National University of Singapore. From 2018 to 2019, he was a Lecturer at the School of Electrical and Information Engineering, the University of Sydney, Australia.

Dr. Zeng is recognized as 2019 Highly Cited Researcher by Web of Science Group. He is the recipient of the Australia Research Council (ARC) Discovery Early Career Researcher Award (DECRA), 2020 IEEE Marconi Prize Paper Award in Wireless Communications, 2018 IEEE Communications Society Asia-Pacific Outstanding Young Researcher Award, 2020 \& 2017 IEEE Communications Society Heinrich Hertz Prize Paper Award. He serves as an Associated Editor for IEEE Communications Letters and IEEE Open Journal of Vehicular Technology, Leading Guest Editor for IEEE Wireless Communications on "Integrating UAVs into 5G and Beyond" and China Communications on "Network-Connected UAV Communications". He is the workshop co-chair for ICC 2018/2019/2020 workshop on UAV communications, the tutorial speaker for Globecom 2018/2019 and ICC 2019 tutorials on UAV communications.

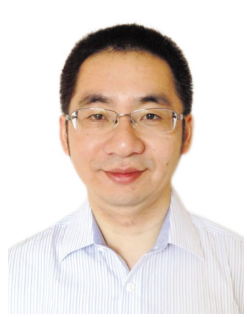

Shi Jin (S'06-M'07-SM'17) received the B.S. degree in communications engineering from Guilin University of Electronic Technology, Guilin, China, in 1996, the M.S. degree from Nanjing University of Posts and Telecommunications, Nanjing, China, in 2003, and the Ph.D. degree in information and communications engineering from Southeast University, Nanjing, in 2007.

From June 2007 to October 2009, he was a Research Fellow with the Adastral Park Research Campus, University College London, London, U.K. $\mathrm{He}$ is currently with the Faculty of the National Mobile Communications Research Laboratory, Southeast University. His research interests include spacetime wireless communications, random matrix theory, information theory, intelligent communications, and intelligent surfaces.
Dr. Jin and his co-authors have been awarded the 2011 IEEE Communications Society Stephen O. Rice Prize Paper Award in the field of communication theory and a 2010 Young Author Best Paper Award by the IEEE Signal Processing Society. He serves as an Associate Editor for the IEEE Transactions on Wireless Communications, the IEEE Communications Letters, and IET Communications.

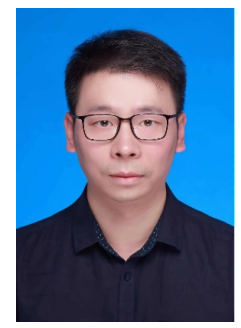

Qiang Cheng (M'15) received the B.S. and M.S degrees from the Nanjing University of Aeronautics and Astronautics, Nanjing, China, in 2001 and 2004, respectively, and the Ph.D. degree from Southeast University, Nanjing, in 2008.

In 2008, he joined the State Key Laboratory of Millimeter Waves, Southeast University, where he was involved in the development of metamaterials and metadevices. He is currently a Full Professor with the School of Information Science and Engineering, Southeast University. He leads a group of Ph.D. students and master students in the area of metamaterials, tunable microwaves circuits, microwave imaging, and terahertz systems. He has authored or coauthored more than 100 publications, with citation over 2000 times.

Dr. Cheng was a recipient of the 2010 Best Paper Award from the New Journal of Physics, the China's Top Ten Scientific Advances of 2010, and the Second Class National Natural Science Award in 2014. He has served as the Vice Chair for the 2008 and 2010 International Workshop on Metamaterials.

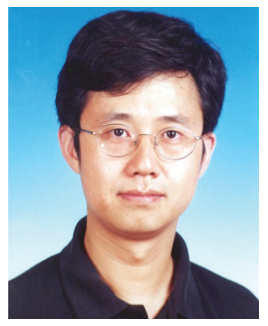

Tie Jun Cui (M'98-SM'00-F'15) received the B.Sc., M.Sc., and Ph.D. degrees in electrical engineering from Xidian University, Xi'an, China, in 1987, 1990, and 1993, respectively.

In March 1993, he joined the Department of Electromagnetic Engineering, Xidian University, and was promoted to an Associate Professor in November 1993. From 1995 to 1997, he was a Research Fellow with the Institut fur Hochstfrequenztechnik und Elektronik (IHE), University of Karlsruhe, Karlsruhe, Germany. In July 1997, he joined the Center for Computational Electromagnetics, Department of Electrical and Computer Engineering, University of Illinois at Urbana-Champaign, Urbana, IL, USA, first as a Post-Doctoral Research Associate and then a Research Scientist. In September 2001, he joined the Department of Radio Engineering, Southeast University, Nanjing, China, as a Cheung-Kong Professor, where he became a Chief Professor in January 2018. He has authored books titled Metamaterials - Theory, Design, and Applications (Springer, November 2009) and Metamaterials: Beyond Crystals, Noncrystals, and Quasicrystals (CRC Press, March 2016). He has authored over 500 peer-reviewed journal articles, which have been cited by more than 25000 times (H-factor 79; from Google Scholar), and holds over 60 patents.

Dr. Cui was awarded a Research Fellowship from the Alexander von Humboldt Foundation, Bonn, Germany, in 1995, the Young Scientist Award from the International Union of Radio Science in 1999, a Cheung Kong Professor under the Cheung Kong Scholar Program by the Ministry of Education, China, in 2001, the National Science Foundation of China for Distinguished Young Scholars in 2002, the Natural Science Award (First Class) from the Ministry of Education, China, in 2011, and the National Natural Science Awards of China (Second Class), in 2014 and 2018, respectively. His research has been selected as one of the most exciting peer-reviewed optics research "Optics in 2016" by Optics and Photonics News Magazine (OSA), 10 Breakthroughs of China Science in 2010, "Best of 2010" in the New Journal of Physics, and Research Highlights in a series of journals. His work has been widely reported by Nature News, MIT Technology Review, Scientific American, Discover, and New Scientists. He has served as a General Co-Chair of the International Workshops on Metamaterials (META'2008, META'2012), a TPC Co-Chair of the Asian-Pacific Microwave Conference (APMC'2005) and the Progress in Electromagnetic Research Symposium (PIERS'2004), and a TPC Member of the International Congress on Advanced Electromagnetic Materials in Microwaves and Optics (Metamaterials'2014, 2016, 2018, 2019). He presented more than 30 keynote and plenary talks in academic conferences, symposiums or workshops. He was an Associate Editor of the IEEE TRANSACTIONS ON GEOSCIENCE AND REMOTE SENSING, and a Guest Editor of Science China - Information Sciences, Science Bulletin, and Research. 